\title{
FTIR analysis of the organics in IDPs: Comparison with the IR spectra of the diffuse interstellar medium
}

\author{
G. Matrajt ${ }^{1}$, G. M. Muñoz Caro ${ }^{1}$, E. Dartois ${ }^{1}$, L. d'Hendecourt ${ }^{1}$, D. Deboffle ${ }^{1}$, and J. Borg ${ }^{1}$ \\ Institut d'Astrophysique Spatiale, Bât. 121, Université Paris-Sud, 91405 Orsay Cedex, France \\ e-mail: graciela.matrajt@ias.u-psud.fr
}

Received 7 July 2004 / Accepted 3 December 2004

\begin{abstract}
We have analyzed seven interplanetary dust particles (IDPs) using an infrared microscope with the aim of comparing the $3.4 \mu \mathrm{m}$ feature to the same feature observed in the diffuse interstellar medium (DISM) and of searching for additional absorption features due to organic matter. We have developed an in situ extraction method based on the etching of the silicate phases with hydrofluoric acid, which allowed a better detection of the organic fraction. Six out of the seven particles studied present a well resolved $3.4 \mu \mathrm{m}$ feature corresponding to aliphatic chains. Some of these particles also present a carbonyl group around $1700 \mathrm{~cm}^{-1}$ due to ketone and, probably also, carboxylic acid compounds. Here we report the first determination of the column densities of the aliphatic and aromatic fractions in IDPs, as well as other functional groups. With the asymmetric $\mathrm{CH}_{3}$ and $\mathrm{CH}_{2}$ column densities we calculated the $\mathrm{CH}_{2} / \mathrm{CH}_{3}$ ratios of the IDPs. The average ratio $\left(\mathrm{CH}_{2} / \mathrm{CH}_{3}=3.7 \pm 0.9\right)$ is clearly larger than the one measured for the DISM (2.2), indicating that the aliphatic chains in IDPs are longer (or less ramified) than in the DISM. The $3.4 \mu \mathrm{m}$ feature of IDPs is significantly narrower than in the DISM, which suggests that the aliphatic fraction in IDPs is made of relatively simpler and less ramified compounds. We conclude that the carbonaceous compounds found in these IDPs have been formed or evolved in a different environment from the DISM.
\end{abstract}

Key words. interplanetary medium - ISM: molecules - astrochemistry - astrobiology - infrared: ISM

\section{Introduction}

Interplanetary dust particles (IDPs) are extraterrestrial particles, also called cosmic dust, collected in the stratosphere by high-altitude aircrafts of the collection facility established by NASA. These particles are composed of different mineral phases, mainly silicates, and by a carbonaceous material which frequently forms the matrix in which the mineral grains are distributed (Brownlee 1978; Bradley et al. 1984).

Several infrared investigations of IDPs and the comparison of their spectra to infrared astrophysical data have been done in the past (Sandford \& Walker 1985; Bradley et al. 1999; Molster et al. 2001; Keller et al. 2002; Molster et al. 2003; Keller \& Flynn 2003). Infrared investigation of the carbonaceous matter in IDPs has also been performed (Flynn et al. 1998, 2002, 2003 , 2004). In particular, Flynn et al. $(1998,2002)$ showed the evidence for the first time for the presence of aliphatic hydrocarbons and of a ketone group, by detecting with an FTIR microscope the $\mathrm{CH}_{2}, \mathrm{CH}_{3}$ and $\mathrm{C}=\mathrm{O}$ stretching functional groups in acid-etched IDPs. Also, Flynn et al. (2003) measured the $\mathrm{CH}_{2} / \mathrm{CH}_{3}$ ratio in several IDPs and compared it to the DISM.

In this work we study quantitatively the $3.4 \mu \mathrm{m}$ aliphatic feature of IDPs, as well as other absorption features due to functional groups in organics (in particular the carbonyl $(\mathrm{C}=\mathrm{O})$ and the $\mathrm{OH}$ stretching features), by examining seven IDPs using an FTIR microscope. We also give a constraint on the abundance of aromatic compounds in IDPs. We first examine their silicate component. Then we dissolve the silicates using hydrofluoric acid and we re-examine the particle after the acidetching to study the organic residue. The results are discussed on the basis of the comparison of the organic feature at $3.4 \mu \mathrm{m}$ of IDPs with the $3.4 \mu \mathrm{m}$ feature observed in the DISM.

\section{Materials and methods}

\subsection{Samples and sample preparation}

We analyzed 7 IDPs. Four of them (L2021-K2; L2011-F2; L2021-K3; L2036-E19) were provided by the NASA-JSC curation facility. The first three originate from "clusters", that formed each from a single fluffy particle that broke up into fragments upon impact on the collector surface, and are therefore known as cluster particles. L2011-F2 belonged to cluster 22, L2021-K2 belonged to cluster 4 and L2021-K3 belonged to cluster 5. L2036-E19 is not a cluster particle.

The other three IDPs (W7116B-U; W7116B-N and W7116B-Y) were extracted from one of the collectors, named W7116, carried by the NASA aircraft. The collector was examined under an optical microscope in a laminar-flow 
10000 -class clean room. Black particles were removed using glass needles and paint brush hairs mounted in a micromanipulator. Because the collectors are coated with silicone oil to better decelerate the particles hitting the surface, the extracted particles are usually covered with silicone oil. Therefore, we rinsed them with hexane (purchased from Aldrich) several times in order to remove the excess of silicone oil. We then transferred them to a mount to be further characterized by Scanning Electron Microscopy (SEM). Energy-Dispersive $\mathrm{X}$-ray (EDX) analyses were performed for each particle using a $20 \mathrm{kV}$ accelerating voltage Jeol 35C microscope equipped with a Voyager $4 \mathrm{X}$-ray analysis system with ThermoNoran light element X-ray detector. The EDX data (not shown) were used to select chondritic particles, since it has been determined that "all stratospheric particles satisfying the chondritic composition criteria are extraterrestrial" (Sandford \& Walker 1985; Bradley et al. 1988).

\subsection{Hydrofluoric acid (HF) treatment}

IDPs are dominated by anhydrous and hydrated silicates, spinnels, carbonates, oxides and sulfides (Fraundorf 1981; Bradley et al. 1988) and a few percent of organic matter, such as PAHs (Clemett et al. 1993, 1999) and aliphatic compounds (Flynn et al. 2003, 2004). Some of the constituent minerals have strong absorption in the infrared region $650-4000 \mathrm{~cm}^{-1}$, which may interfere with the detection of the organic features. In some regions of the spectrum they even mask them. To remove most of this interference, we have developed a micrometer scale etching technique that removes in situ most of the silicate fraction, carbonates and sulfides, preserving the acid-insoluble organic matter. This technique, which is based on the hydrofluoric acid dissolution methodology used to prepare acid insoluble organic residues on meteorites (such as has been done in Orgueil or Murchison (Smith \& Kaplan 1970; Robert \& Epstein 1982; Gardinier et al. 2000)), is similar to the technique developed by Brownlee et al. (2000) and used on micro-scales by Flynn et al. (2002, 2004) for the analysis of organics in IDPs. However, the main difference with this methodology is that in our procedure we perform the etching in situ and over the entire particle, allowing therefore the analysis of the organics present throughout the whole volume of the particle.

The HF attack is performed as follows: after careful hexane cleaning of all the samples, the particles were crushed, following the methodology described by Raynal et al. (2000), on a KRS-5 (Thallium Bromoiodide) window. This type of window is water-insoluble and does not dissolve in HF (whereas the frequently used $\mathrm{KBr}$ does). A first infrared analysis is performed on each sample to determine the kind of silicates present in the particle and therefore its type. Then, each sample is exposed for a few hours to a hydrofluoric acid drop (48 wt\% in water, 99.99\%, purchased from Aldrich) in an Ar or $\mathrm{N}_{2}$ atmosphere to avoid oxidation. This technique allows the removal of most (sometimes all) of the silicates present in the particle very efficiently (Brownlee et al. 2000; Flynn et al. 2002) and does not perturb or modify the organics that are acid-insoluble (Robert \& Epstein 1982; Gardinier et al. 2000). However, it is known that acid hydrolysis breaks peptidic bonds in proteins and dissociates certain organic molecules such as amines. Therefore, we studied the possible effects of HF treatment on several organic standards which were relevant for the identification of the features due to organics in IDPs, and we found no significant changes in the band shapes and positions (Sect. 3.3)

\subsection{Analytical techniques}

\subsubsection{FTIR}

We used Fourier Transform InfraRed spectroscopy, employing a Nicolet Magna-IR 560 ESP spectrometer attached to a Nicolet Nicplan infrared microscope, in line with a Synchrotron Radiation Source located on line SU5 at the Laboratoire pour l'Utilisation du Rayonnement Electromagnétique (LURE) at the University of ParisSud, Orsay, France. The spectrometer is equipped with a $\mathrm{KBr}$ beamsplitter and a nitrogen-cooled mercury-cadmiumtellure (MCT) detector working in the 4000 to $650 \mathrm{~cm}^{-1}(2.5$ to $15 \mu \mathrm{m})$ spectral range. The microscope uses a SchwarzchildCassegrain objective $(32 \times)$ and a condenser $(10 \times)$ in the transmission mode. Besides the Synchrotron light source, the standard light source of the instrument, a conventional black body-like source (Globar) is available. Further details about this instrument and the advantages of both light sources can be found in Raynal et al. (2000). For apertures above $6 \mu \mathrm{m}^{2}$, no significant differences were observed between the spectra obtained with the internal Globar source and the Synchrotron source. Moreover, the Globar was more stable than the Synchrotron source. Therefore, most of the analyses described here were performed using the internal Globar source, more frequently available.

Standards of organic compounds were used to better identify the carrier of the peak around $1700 \mathrm{~cm}^{-1}$. They were all purchased from Aldrich. We used a ketone $\left[\mathrm{R}-\mathrm{C}(=\mathrm{O})-\mathrm{R}^{\prime}\right]$ in solid state at room temperature, 2-hexadodecanone $\left(\mathrm{C}_{16} \mathrm{H}_{32} \mathrm{O}\right)$, and three carboxylic acids [R-COOH]: stearic acid $\left(\mathrm{C}_{18} \mathrm{H}_{36} \mathrm{O}_{2}\right)$, adipic acid $\left(\mathrm{C}_{6} \mathrm{H}_{10} \mathrm{O}_{4}\right)$ and succinic acid $\left(\mathrm{C}_{4} \mathrm{H}_{6} \mathrm{O}_{4}\right)$.

\subsubsection{Baseline correction}

In order to enhance the signal-to-noise ratio and minimize background fluctuations due to purge variations, 25 acquisitions of 256 scans each at the resolution of $2 \mathrm{~cm}^{-1}$ were recorded for each sample and then averaged. However, the baseline in each spectrum is not always easy to establish because it varies according to the thickness of the sample. For example, when the sample is too thick, the IR radiation suffers diffraction, producing a steep decrease in the baseline around the $1000 \mathrm{~cm}^{-1}$ region (see for an example the spectrum of particle W7116B-Y in Fig. 1). The baseline also varies according to the type of material the IR beam passes through, and for silicates the baseline is hardly horizontal (see particle L2021-K3 in Fig. 2 for a representative example). We have corrected our spectra by subtracting a spline baseline. The baseline is the result of a spline fit, generated by an in-house IDL program, to a number of points given manually. Our code also provides the 
ORIGINAL
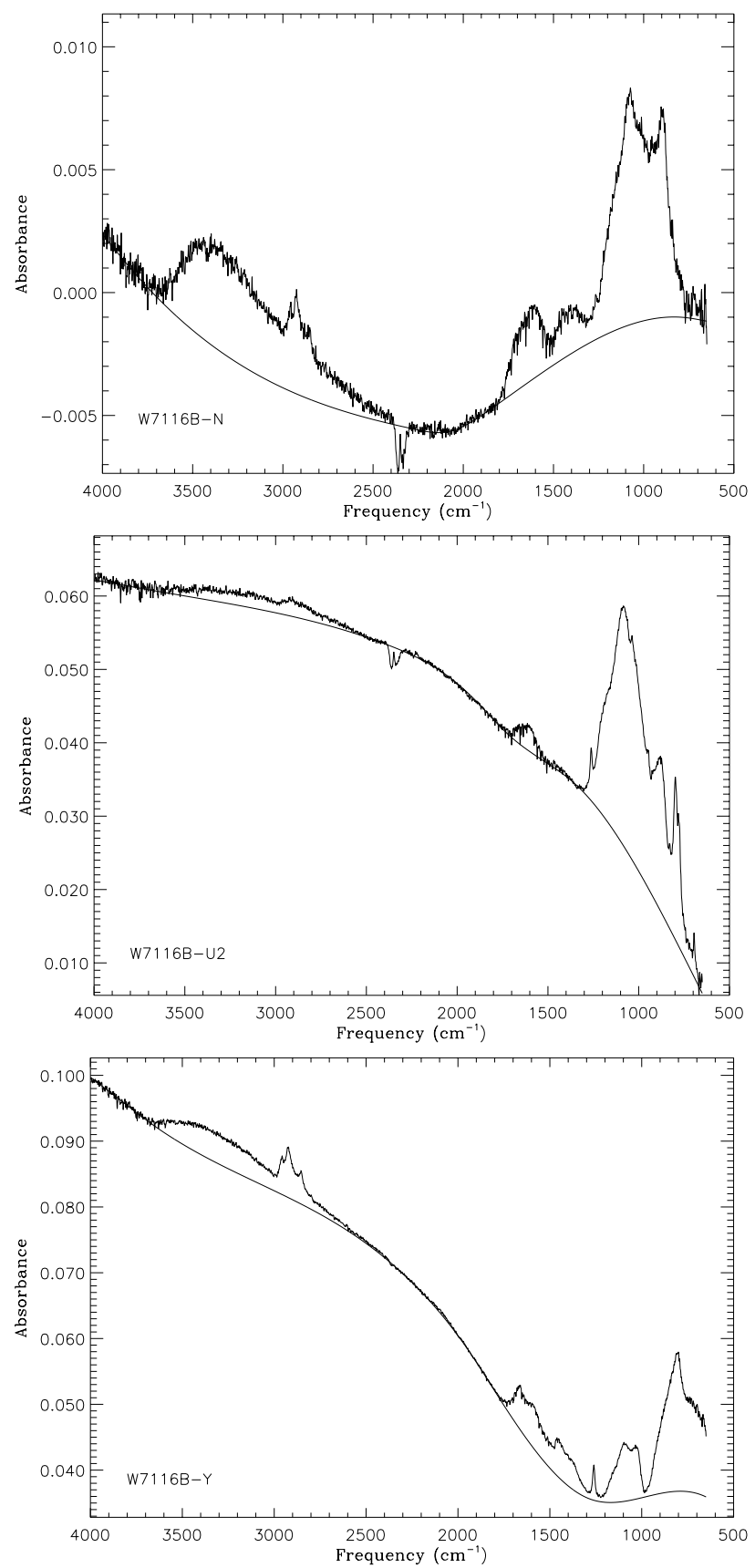

BASE LINE CORRECTED
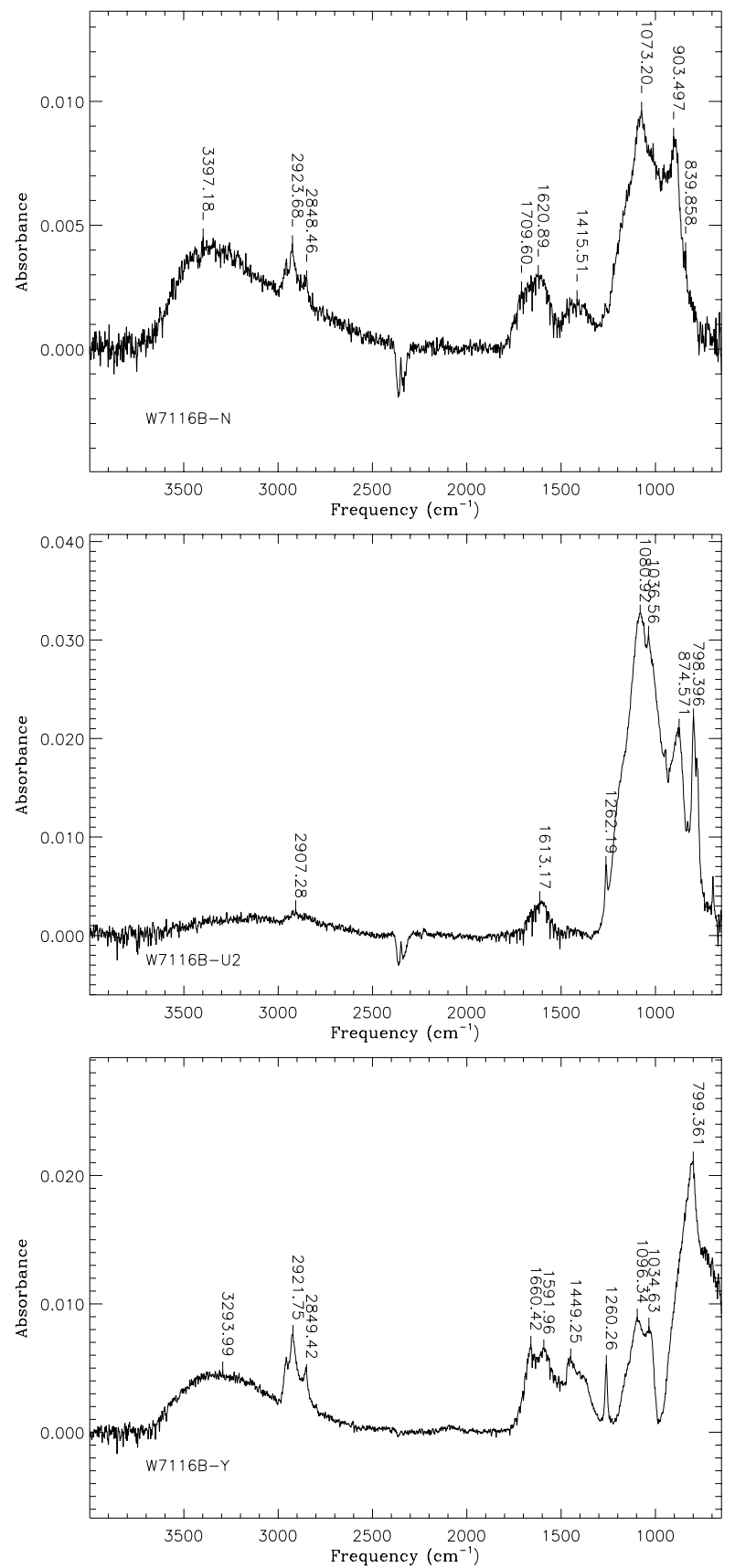

Fig. 1. Spectra of the W7116B particles studied before HF dissolution. The left column shows the original spectra with the corresponding baseline, the right column shows the same spectra after baseline subtraction.

positions and areas of the spectral features. The original spectra of the IDPs are presented in Figs. 1, 2, 4 and 5: the panels in the left column show the original spectra with the corresponding baseline and the panels in the right column are the spectra after baseline subtraction.

\section{Results}

Figures 1 and 2 show the spectra of all the particles analyzed during this study before the HF etching. The spectra of the same particles after HF treatment are shown in Figs. 4 and 5.

\subsection{FTIR analyses before HF dissolution}

Many particles (W7116B-U, W7116B-Y, L2011-F2, L2021-K3, L2021-K2 and L2036-E19) present remaining silicone oil, with characteristic peaks at 806-800, 1095-1080, 1260, $2963 \mathrm{~cm}^{-1}$ (see the silicone oil spectrum in Fig. 8). The particle W7116B-Y has also peaks at $1457-1450 \mathrm{~cm}^{-1}$, corresponding to carbonates. There are also some particles (W7116B-N, L2011-F2 and L2021-K3) with peaks near $\sim 1400 \mathrm{~cm}^{-1}$ whose interpretation is at present unknown. In Table 1 all the peaks observed before the HF dissolution 
ORIGINAL
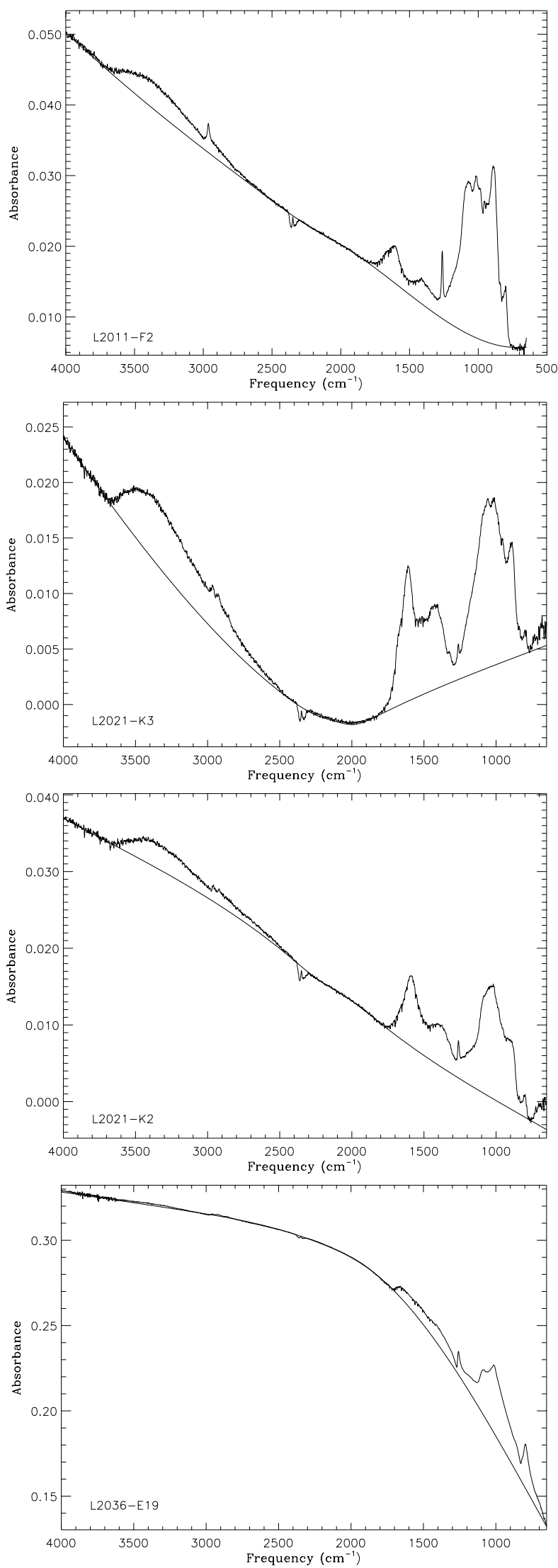

BASE LINE CORRECTED
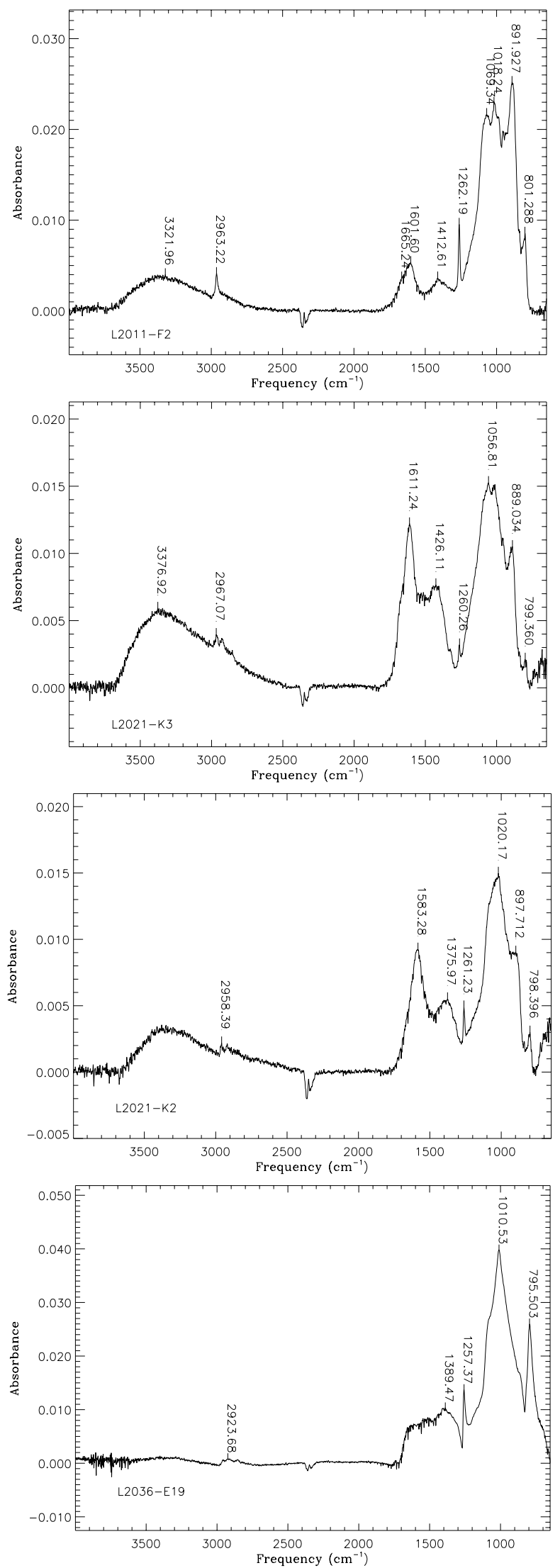

Fig. 2. Spectra of the NASA particles studied before HF dissolution. The left column shows the original spectra with the corresponding baseline, the right column shows the same spectra after baseline subtraction. 
Table 1. Peaks present in the spectra before the HF treatment.

\begin{tabular}{|c|c|c|c|}
\hline $\begin{array}{c}\text { Peak position } \\
\left(\mathrm{cm}^{-1}\right)\end{array}$ & Vibration mode & Carrier & Interpretation \\
\hline $794-780$ & Si-O-Si bending & silicate & phyllosilicate \\
\hline $806-800$ & $\mathrm{Si}-\mathrm{O}-\mathrm{Si}$ bending & siloxane & remaining silicone oil ${ }^{a}$ \\
\hline $840-837$ & Si-O stretching & silicate & olivine $^{a}$ \\
\hline $890-880$ & " & silicate & olivine $^{a}$ \\
\hline $955-950$ & Si-O stretching & silicate & pyroxene $^{a}$ \\
\hline $990-970$ & " & silicate & olivine $^{a}$ \\
\hline $1014-1010$ & $"$ & silicate & pyroxene $^{a}$ \\
\hline 1018 & $"$ & silicate & phyllosilicate $^{a}$ \\
\hline $1070-1060$ & Si-O stretching & silicate & pyroxene $^{a}$ \\
\hline $1095-1080$ & Si-O stretching & siloxane & remaining silicone oil ${ }^{a}$ \\
\hline 1260 & Si-O stretching & siloxane & remaining silicone oil ${ }^{a}$ \\
\hline 1375 & $\mathrm{COO}^{-}$sym stretch & organic & carbox. acid salts \\
\hline $1415-1400$ & & & unknown \\
\hline $1457-1450$ & $\mathrm{CO}_{3}^{-}$ & carbonate ion & carbonates \\
\hline 1583 & $\mathrm{COO}^{-}$antisym stretch & organic & carbox. acid salts \\
\hline $1667-1660$ & $\mathrm{H}-\mathrm{O}-\mathrm{H}$ bending & water & phyllosilicate \\
\hline $3000-2800$ & $\mathrm{CH}_{2} ; \mathrm{CH}_{3}$ sym and asym stretch & organic & aliphatic hydrocarbons ${ }^{a}$ \\
\hline 2963 & Si-O stretching & siloxane & remaining silicone oil ${ }^{a}$ \\
\hline $3600-3100$ (centered at 3400) & $\mathrm{OH}$ stretch & water & water in phyllosil., carbox. acids, alcohols \\
\hline
\end{tabular}

${ }^{a}$ Unambiguous assignment.

are listed, together with their ascribed vibration mode and assignment.

\subsubsection{Identification of silicates}

The analyses of the particles before HF dissolution make it possible to see the silicate signature of each IDP. Sandford \& Walker (1985) described FTIR analyses of several IDPs and found that IDPs can be grouped in three spectral classes referred to as olivines (Oli), layer-lattice silicates (LLS) and pyroxenes $(\mathrm{Px})$ after the minerals that provide the best match to the dust spectra. Following this description, we have compared each one of the spectra obtained during our work with the representative spectra of each of the three classes established by Sandford \& Walker (1985), and classified all the particles studied (Table 2). From this classification we found that four out of seven particles (W7116B-Y; W7116B-N; L2021-K3 and L2021-K2) seem to have a mixture of anhydrous and hydrated layer-lattice silicates, being therefore hydrated IDPs. The remaining particles are composed of anhydrous minerals, either only pyroxene (L2036-E19) or a mixture of olivine and pyroxene (W7116B-U and L2011-F2), and thus are anhydrous IDPs.

\subsubsection{Identification of organic peaks}

Besides the peaks related to silicates, most of the particles also display peaks related to organic compounds that are frequently found in two main regions: $3000-2800 \mathrm{~cm}^{-1}$ (aliphatic $\mathrm{C}-\mathrm{H}$ stretching modes) and $1700-1300 \mathrm{~cm}^{-1}$ (the assignment of these is difficult due to the presence of water in phyllosilicates $(\mathrm{OH}-$ bending mode) and carbonate features in the same region). In the region $3000-2800 \mathrm{~cm}^{-1}$ four main peaks are visible which correspond to the $\mathrm{CH}_{2}$ and $\mathrm{CH}_{3}$ symmetric and asymmetric stretching peaks at about 2850, 2865, 2920 and $2958 \mathrm{~cm}^{-1}$ (Fig. 3). Most of the particles show only three

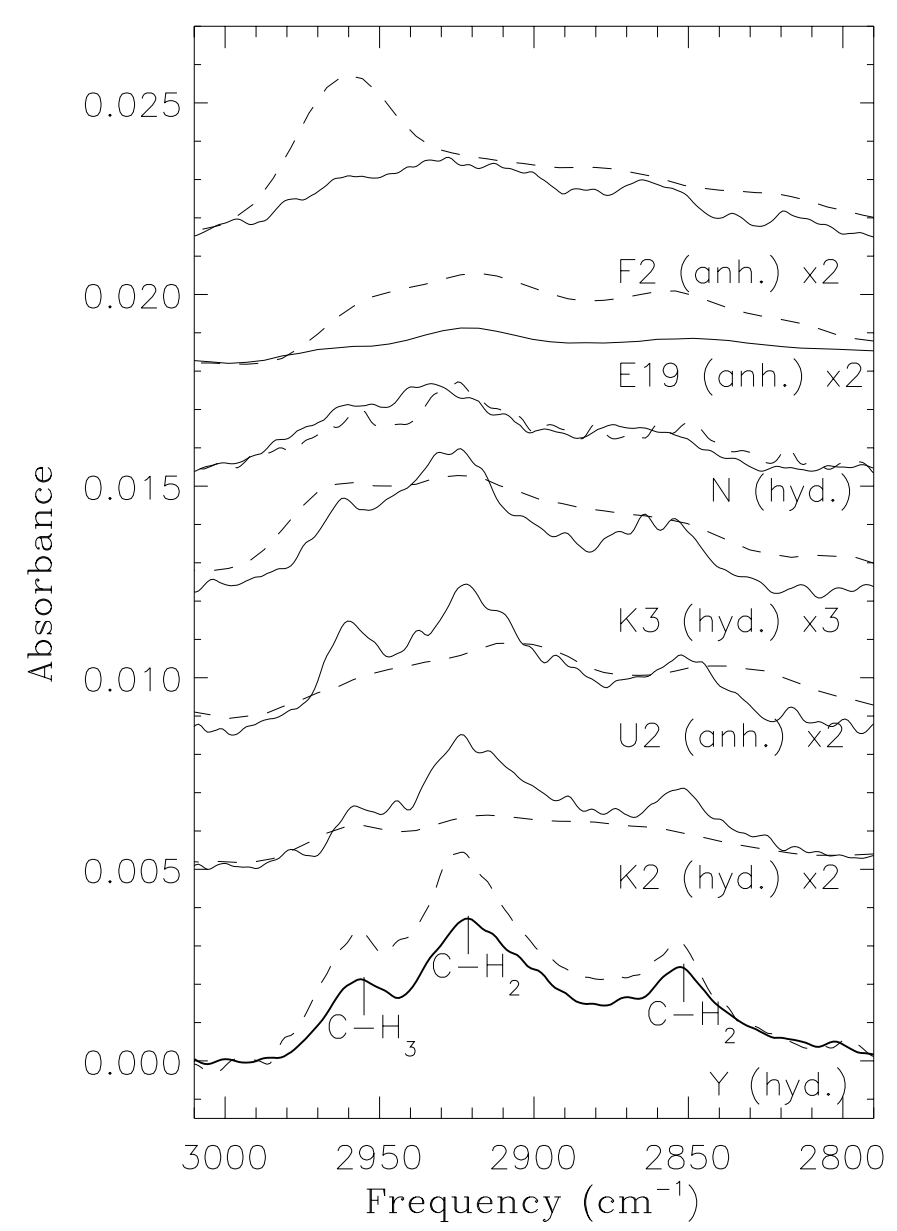

Fig. 3. The aliphatic peaks of the region $3000-2800 \mathrm{~cm}^{-1}$, for the seven IDPs before (dashed line) and after (solid line) HF treatment. Some spectra have been multiplicated by a factor (indicated in the right side) for intercomparison. 
Table 2. IDPs classification.

\begin{tabular}{|c|c|c|c|}
\hline Name & Type & Silicate class $^{a}$ & main silicate peaks $\left(\mathrm{cm}^{-1}\right)$ \\
\hline W7116B-Y & chondritic & LLS+Oli+Px (hydrated) & $1030 ; 1460 ; 1660$ \\
\hline W7116B-N & chondritic & LLS+Px+Oli (hydrated) & $895 ; 1010 ; 1075 ; 1380 ; 1450 ; 1620$ \\
\hline W7116B-U & chondritic & Px+Oli (anhydrous) & 770; 790; 880; 950; 1030; 1074; 1660 \\
\hline L2011-F2 & cluster & $\mathrm{Px}+\mathrm{Oli}$ (anhydrous) & 840; 890; 990; 1017; 1069; 1600 \\
\hline L2036-E19 & chondritic & Px (anhydrous) & $794 ; 1011$ \\
\hline L2021-K3 & cluster & LLS+Px+Oli (hydrated) & $888 ; 955 ; 1012 ; 1056 ; 1606 ; 1324 ; 3500$ \\
\hline L2021-K2 & cluster & LLS+Oli (hydrated) & $758 ; 837 ; 890 ; 1018$ \\
\hline
\end{tabular}

${ }^{a}$ LLS = layer lattice silicate. Oli $=$ olivine. $\mathrm{Px}=$ pyroxene.

Table 3. Band strengths of molecules used for integration.

\begin{tabular}{cccccc}
\hline \hline Species & Vibration mode & $\begin{array}{c}\text { Position } \\
\mu \mathrm{m}\end{array}$ & $\begin{array}{c}\text { Position } \\
\mathrm{cm}^{-1}\end{array}$ & $\begin{array}{c}A \\
\mathrm{~cm} \mathrm{group}^{-1}\end{array}$ & Ref. \\
\hline silicates & $\mathrm{SiO}$ str. & 10 & $\sim 1000$ & $2.0 \times 10^{-16}$ & $a$ \\
aliphatics & $\mathrm{CH}$ str. & 3.4 & $2800-3000$ & $1.2 \times 10^{-17}$ & $b$ \\
$\mathrm{CH}_{2}$ group & asym. $\mathrm{CH}_{2}$ str. & 3.42 & 2922 & $8.4 \times 10^{-18}$ & $c$ \\
$\mathrm{CH}_{3}$ group & asym. $\mathrm{CH}_{3}$ str. & 3.38 & 2958 & $1.25 \times 10^{-17}$ & $c$ \\
ketones & $\mathrm{C}=\mathrm{O}$ str. & 5.83 & 1715 & $1.2 \times 10^{-17}$ & $d$ \\
carboxylic acids & $\mathrm{C}=\mathrm{O}$ str. & 5.88 & 1700 & $3.2 \times 10^{-17}$ & $d$ \\
carboxylic acids & $\mathrm{OH}$ str. & $2.77-4.35$ & $3600-2300$ & $2.8 \times 10^{-16}$ & $e$ \\
carboxylic acid salts & $\mathrm{COO}^{-}$str. & 6.3 & 1586 & $6.0 \times 10^{-17}$ & $d$ \\
aromatics & ring stretch & 6.2 & 1600 & $2 \times 10^{-19}$ & $f$ \\
\hline
\end{tabular}

${ }^{a}$ Jaeger et al. (1998), in cm (SiO bond $)^{-1}$.

${ }^{b}$ Dartois et al. (2004), in $\mathrm{cm}(\mathrm{C} \text { atom })^{-1}$, based on the value for hexane $7.2 \times 10^{-17} \mathrm{~cm} \mathrm{molec}^{-1}$ dividing by 6 for the number of $\mathrm{C}$ atoms (see text for details).

${ }^{c}$ Dartois et al. (2004), in cm (C atom $)^{-1}$.

${ }^{d}$ Wexler (1967).

${ }^{e} \mathrm{Using}$ area(OH str.)/area(C=O str.) and for glycolic acid (carboxylic acid) we obtain $A(\mathrm{OH}) \approx 2.8 \times 10^{-16}$.

${ }^{f}$ Schutte et al. (1998), in $\mathrm{cm}(\mathrm{C} \text { atom })^{-1}$, corresponding to the average of neutral PAHs.

prominents peaks, since the $\mathrm{CH}_{3}$ symmetric vibration, which is the weakest band, is blended with the $\mathrm{CH}_{2}$ band at $2850 \mathrm{~cm}^{-1}$. The particle W7116B-U does not present distinguishable peaks but rather a broad band. The particle L2011-F2 does not present any of these four peaks; instead it displays a peak at $2960 \mathrm{~cm}^{-1}$, which is not organic but a remnant of the silicone oil. Moreover, the L2021-K2 particle presents peaks at 1375 and $1583 \mathrm{~cm}^{-1}$ which can be related to the $\mathrm{COO}^{-}$stretch of a carboxylic acid salt (see Sect. 3.4).

In Table 4 are shown the areas of the features corresponding to the $\mathrm{Si}-\mathrm{O}$ stretch of silicates (between $1100-700 \mathrm{~cm}^{-1}$, "10 $\mu \mathrm{m}$ band") and the $\mathrm{C}-\mathrm{H}$ stretch of aliphatics (between $3000-2800 \mathrm{~cm}^{-1}$, "3.4 $\mu \mathrm{m}$ band") that were calculated after a baseline correction of each spectrum. It should be noted that the exact values of the areas calculated are baseline dependent, but are evaluated in the same way for each IDP, allowing intercomparison. The estimated error of the area is calculated by comparing the result obtained with a spline baseline (shown in Fig. 1) with the result using a linear baseline for the local continuum. The error so obtained is less than $30 \%$ of the calculated area for the broad $\mathrm{OH}$ stretch and no more than $15 \%$ for the other bands. The column densities for aliphatics $(N[3.4]$, in aliphatic $\mathrm{C}$ atoms $\left./ \mathrm{cm}^{2}\right)$ and silicates $\left(N[10]\right.$, in $\mathrm{Si}$ atoms $\left./ \mathrm{cm}^{2}\right)$ and the ratio $N[3.4] / N[10]$, in aliphatic $\mathrm{C}$-atoms/Si-atoms are also given. These column densities were determined using the band strengths for different molecules (see Table 3) and the formula:

$N=\int_{\text {band }} \frac{\tau_{\nu} \mathrm{d} v}{A}$

where $N$ is the column density in $\mathrm{cm}^{-2}, \tau$ the optical depth of the band, and $A$ the band strength in $\mathrm{cm} \mathrm{molec}^{-1}$. Assuming that the carrier of the $3.4 \mu \mathrm{m}$ feature is a pure aliphatic molecule, and using the band strength of the whole $3.4 \mu \mathrm{m}$ feature calculated for hexane ice $\left(7.2 \times 10^{-17} \mathrm{~cm} \mathrm{molec}^{-1}\right.$ (Dartois et al. 2004)) and dividing by six for the number of carbon atoms in the molecule, we have estimated the band strength $(A[\mathrm{C}])$ of the number of $\mathrm{C}$ atoms which contributes to the $\mathrm{C}-\mathrm{H}$ stretching vibration in aliphatics. This estimate is $A[C]=1.2 \times 10^{-17} \mathrm{~cm}$ $(\mathrm{C} \text { atom })^{-1}$. A similar value is obtained if ethane is used as the carrier molecule. Therefore, the value of $A[C]$ is not very dependent on the exact identification of the aliphatic carrier. It is, however, better to calculate the column density of aliphatic $\mathrm{C}$ atoms as $N[\mathrm{C}]=N\left[\mathrm{CH}_{2}\right]+N\left[\left(\mathrm{CH}_{3}\right]\right.$, where the areas of the 
Table 4. Aliphatic column density (noted $N[3.4]$, in the region $3000-2800 \mathrm{~cm}^{-1}$ ) and silicate column density $\left(N[10]\right.$, around $\left.1000 \mathrm{~cm}^{-1}\right)$ of the IDPs before the HF treatment, calculated after baseline correction. See text for error estimates.

\begin{tabular}{cccccc}
\hline \hline IDP & area [3.4] & area [10] & $\begin{array}{c}N[3.4] \\
\left.\text { (aliph C atom/cm } / \mathrm{cm}^{2}\right)\end{array}$ & $\begin{array}{c}N[10] \\
\left(\mathrm{Si} \text { atoms/cm } / \mathrm{cm}^{2}\right)\end{array}$ & $\begin{array}{c}N[3.4] / N[10] \\
\text { (aliph. C atoms/Si atoms) }\end{array}$ \\
\hline W7116B-N & 0.11 & 2.3 & $2.1 \times 10^{16}$ & $2.7 \times 10^{16}$ & 0.78 \\
W7116B-Y & 0.43 & 5.2 & $8.2 \times 10^{16}$ & $6.0 \times 10^{16}$ & 1.4 \\
W7116B-U & 0.07 & 10.0 & $1.3 \times 10^{16}$ & $1.2 \times 10^{17}$ & 0.11 \\
L2036-E19 & 0.11 & 5.0 & $2.1 \times 10^{16}$ & $5.9 \times 10^{16}$ & 0.36 \\
L2021-K3 & 0.12 & 3.6 & $2.3 \times 10^{16}$ & $4.2 \times 10^{16}$ & 0.55 \\
L2021-K2 & 0.05 & 3.0 & $9.6 \times 10^{15}$ & $3.6 \times 10^{16}$ & 0.27 \\
L2011-F2 & 0.14 & 6.0 & $2.7 \times 10^{16}$ & $7.0 \times 10^{16}$ & 0.39 \\
\hline
\end{tabular}

peaks at 2958 and $2922 \mathrm{~cm}^{-1}$ (asymmetric $\mathrm{CH}_{3}$ and $\mathrm{CH}_{2}$ str. modes, see Table 3 ) are calculated with a Gaussian fit. The first method was used for the spectra measured before hydrolysis, since generally the 2958 and $2922 \mathrm{~cm}^{-1}$ bands were not well resolved. The second method was used for the spectra after hydrolysis. It was found that the value of the estimated column density was typically $30 \%$ higher when the first method, less precise, was used.

The band strength of the $\mathrm{SiO}$ bond $(A[\mathrm{SiO}])$ has been determined as follows:

$A[\mathrm{SiO}]=m a^{\mathrm{int}} \times m(\mathrm{SiO})$

where $m(\mathrm{SiO})$ is the molecular mass of one enstatite molecule $\left(100 /\right.$ Avogadro number $\left.=1.66 \times 10^{-22} \mathrm{~g}\right)$, and $m a^{\text {int }}$ is the integrated mass absorption coefficient defined by:

$m a^{\text {int }}=\int \frac{4 \pi n}{\rho \lambda}$

where $n$ is the refraction index (Jaeger et al. 1998), $\lambda$ is the wavelength (Jaeger et al. 1998), and $\rho$ is the density for enstatite $\left(3.3 \mathrm{~g} / \mathrm{cm}^{3}\right)$ (Koike et al. 1993). From the above equations we obtain $A[\mathrm{SiO}]=2.0 \times 10^{-16} \mathrm{~cm}(\mathrm{Si} \text { atoms })^{-1}$. Since the particles were crushed we assumed that the thickness and porosity of the surface being analyzed were constant. The aperture used was chosen to totally cover the particle, and varied from $25 \times 25 \mu \mathrm{m}^{2}$ to $30 \times 30 \mu \mathrm{m}^{2}$.

\subsubsection{Proportion of silicates vs. organics}

The measurements of the column densities of the bands due to the $\mathrm{CH}(N[3.4])$ and $\mathrm{SiO}(N[10])$ stretchings, and their comparison $(N[3.4] / N[10]$, Table 4$)$ shows that the number of Si atoms is larger than the number of $\mathrm{C}$ atoms in these IDPs, with one exception: W7116B-Y. The proportions, however, are not similar, showing that some particles contain more organics than others.

\subsection{IR analyses after HF dissolution}

In Table 5 the main peaks observed after the HF dissolution are shown, together with the vibration mode they may represent and their potential identifications. Figures 4 and 5 show the spectra of the particles after the HF treatment.

Many of the peaks that were present before the $\mathrm{HF}$ treatment have been reduced (or disappeared) after the acid dissolution (790-820; 870-905; 1010-1020; 1050-1100;
$1400-1450 ; 2963 \mathrm{~cm}^{-1}$ ). Moreover, some peaks that were not clearly distinguished before the HF treatment can now be found $\left(1700-1714 ; 1450-1500 ; \sim 1600 ; 3250 \mathrm{~cm}^{-1}\right)$. There are also some peaks that were already present before the HF treatment which are still observed after the acid dissolution $(800-806 ; 1080-1095 ; 1260 ; 1375 ; 1400-1460 ; 1583$; 2800-3000; 3100-3600 $\mathrm{cm}^{-1}$ ). In a general manner, the $\mathrm{HF}$ treatment has dissolved most of the silicate fraction off the particles, as can be seen in Table 6. The column densities for both silicates and aliphatics are also shown in Table 6 . Table 8 reports the areas and column densities of the bands at $3600-2400,1700$, and $1585 \mathrm{~cm}^{-1}$ attributed to the $\mathrm{OH}, \mathrm{C}=\mathrm{O}$ and $\mathrm{COO}^{-}$functional groups. The areas and column densities of the band at $1600 \mathrm{~cm}^{-1}$, assigned to aromatic compounds, are also indicated. These values should be taken only as indicative, as they may present large uncertainties that are caused by the uncertainties in the values of the band strengths, the decomposition of the bands needed for integration, and possible effects due to the HF treatment required for its detection. In the regions where two bands were blended we used a Gaussian decomposition for the integration of the areas.

\subsubsection{Aliphatics in the region $3000-2800 \mathrm{~cm}^{-1}$}

After the HF treatment all particles (except L2036-E19) have three peaks corresponding to the $\mathrm{CH}_{2}$ symmetric vibration (at $\sim 2850 \mathrm{~cm}^{-1}$ ), the $\mathrm{CH}_{2}$ asymmetric vibration (at $\sim 2922 \mathrm{~cm}^{-1}$ ) and the $\mathrm{CH}_{3}$ asymmetric vibration (at $\sim 2958 \mathrm{~cm}^{-1}$ ). The weak $\mathrm{CH}_{3}$ symmetric vibration (at $\sim 2865 \mathrm{~cm}^{-1}$ ), is generally blended with the $\sim 2850 \mathrm{~cm}^{-1}$ band.

The particle L2036-E19 has lost the aliphatic peaks that were present before the HF treatment. The particle W7116B-Y lost part of its aliphatic component, as shown by the decrease of the area of the $3.4 \mu \mathrm{m}$ feature ([3.4] area, Table 6). This decrease in the aliphatic fraction can be explained by the removal of an acid-soluble organic compound whose origin is at present unknown. Because this compound washes out easily with HF, it could also be attributed to an organic contaminant that was sticking on the surface of the particle. Therefore, the excess of organic in these two particles may be considered to be either indigenous or a surface contamination. Particle L2011-F2 presented a prominent peak at $2963 \mathrm{~cm}^{-1}$ due to the remaining silicone oil, which was removed by the HF treatment. 
ORIGINAL
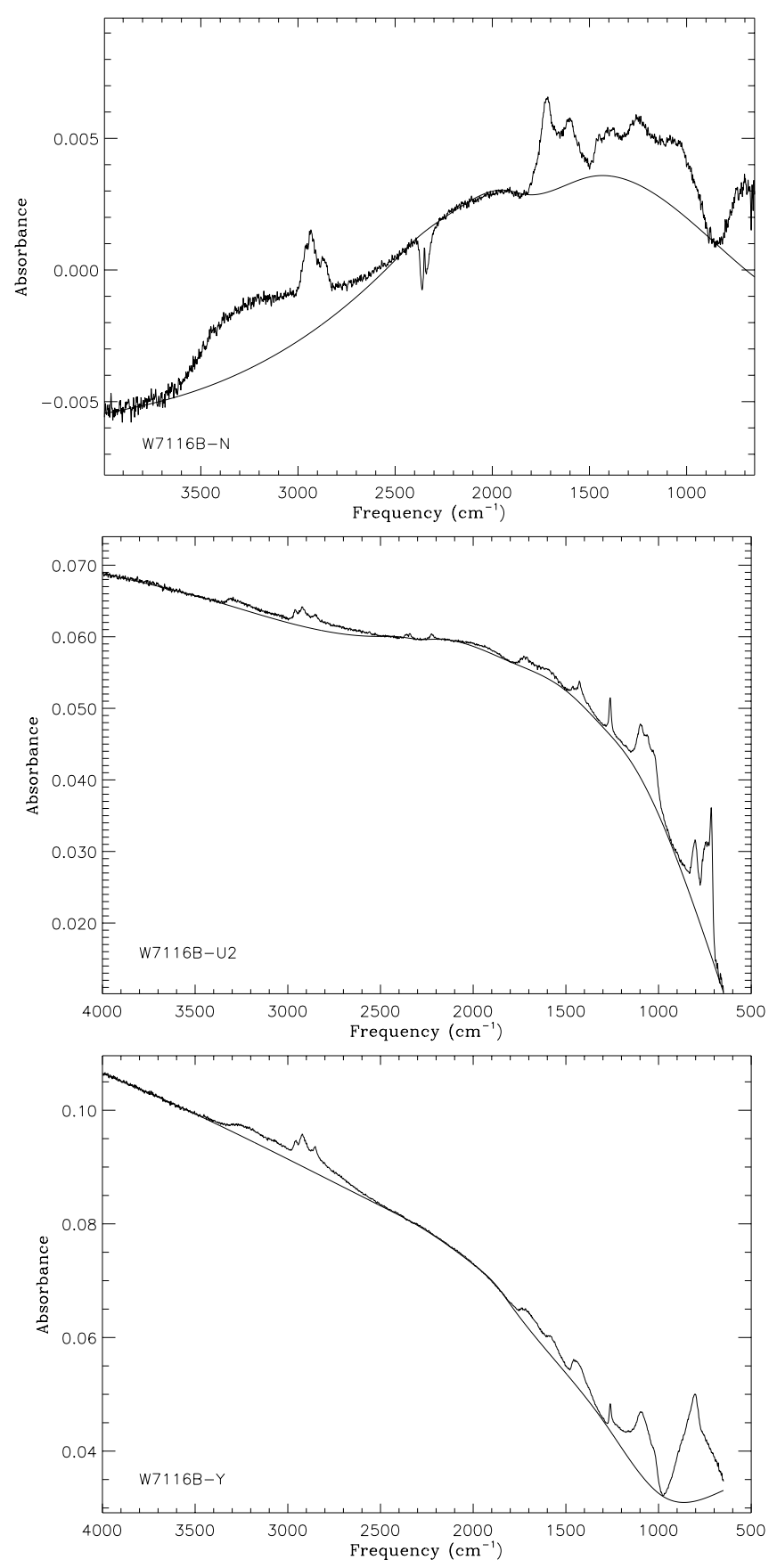

BASE LINE CORRECTED
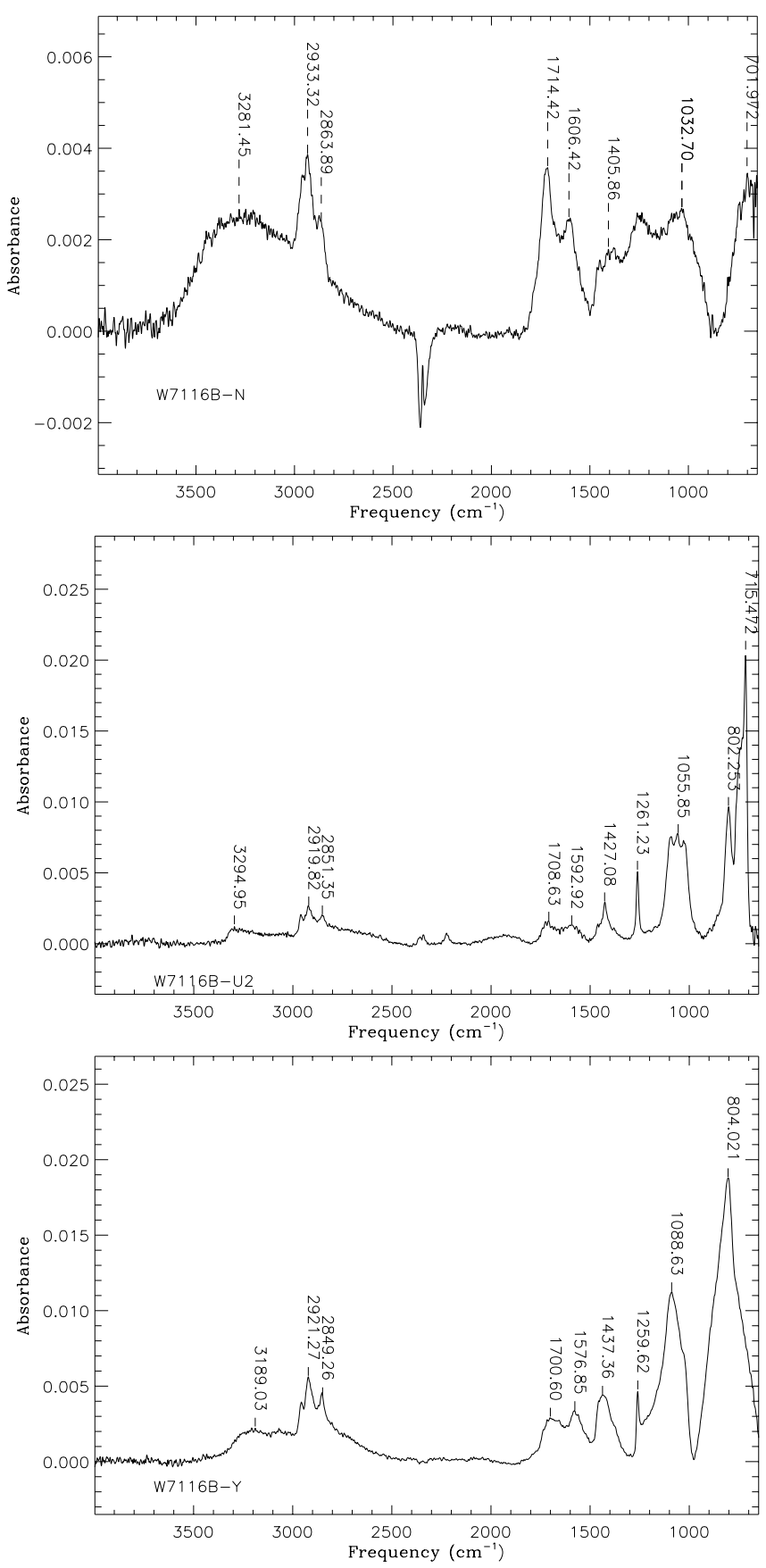

Fig. 4. Spectra of the W7116B particles studied after HF dissolution. The left column shows the original spectra with the corresponding baseline, the right column shows the same spectra after baseline subtraction. Please note the difference in scale with respect to the spectra obtained before HF (Fig. 1).

\subsubsection{The $\mathrm{CH}_{2}$-to- $\mathrm{CH}_{3}$ ratio in IDPs}

The column densities of the $\mathrm{CH}_{3}$ and $\mathrm{CH}_{2}$ groups, $N\left[\mathrm{CH}_{3}\right]$ and $N\left[\mathrm{CH}_{2}\right]$, were calculated by fitting the 2958 and $2922 \mathrm{~cm}^{-1}$ bands with two Gaussians, using the integrated band strengths given in Sect. 3.1.2. The fitting procedure was tested with the spectrum measured toward GC IRS7, a probe of the diffuse interstellar medium, obtaining $N\left[\mathrm{CH}_{2}\right] / N\left[\mathrm{CH}_{3}\right]=2.2$, which is in agreement with the literature value of $2.0-2.5$
(Sandford et al. 1991; Pendleton et al. 1994). The values of $N\left[\mathrm{CH}_{3}\right], N\left[\mathrm{CH}_{2}\right]$, and $N\left[\mathrm{CH}_{2}\right] / N\left[\mathrm{CH}_{3}\right]$, are given in Table 7.

The average ratio $\mathrm{CH}_{2} / \mathrm{CH}_{3}$ calculated for the anhydrous particles is 3.0 and the one calculated for the hydrated particles is 4.1. The difference between these two ratios is probably not significant as the number of IDPs studied remains limited. The shape and bandwidth of the $3.4 \mu \mathrm{m}$ feature is quite similar in both types of IDPs. Moreover, the values of $N\left[\mathrm{CH}_{2}\right]$ and $N\left[\mathrm{CH}_{3}\right]$ do not differ significantly, indicating that 

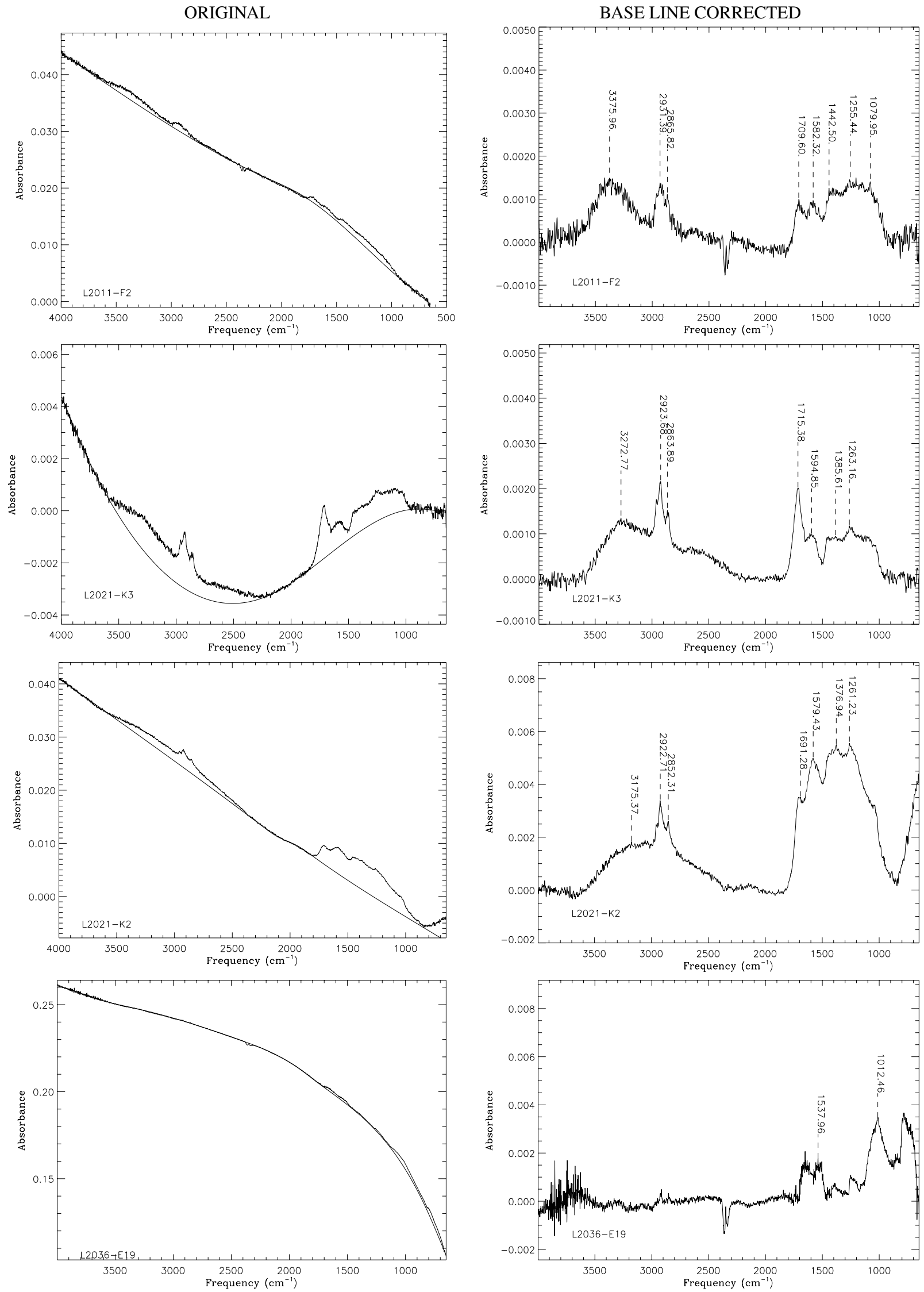

Fig. 5. Spectra of the NASA particles studied after HF dissolution. The left column shows the original spectra with the corresponding baseline, the right column shows the same spectra after baseline subtraction Please note the difference in scale with respect to the spectra obtained before HF (Fig. 2). 
Table 5. Peaks present in the spectra after the HF treatment.

\begin{tabular}{|c|c|c|c|}
\hline $\begin{array}{c}\text { Peak position } \\
\left(\mathrm{cm}^{-1}\right)\end{array}$ & Vibration mode & Carrier & Possible interpretation \\
\hline $806-800$ & $\mathrm{Si}-\mathrm{O}$ & siloxane & remaining silicone oil \\
\hline$\sim 1000$ & Si-O stretching & silicate & remaining silicates \\
\hline $1095-1080$ & Si-O stretch & siloxane & remaining silicone oil \\
\hline 1260 & Si-O stretching & siloxane & remaining silicone oil \\
\hline 1375 & $\mathrm{COO}^{-}$sym stretch & organic & carbox. acid salts \\
\hline $1460-1447$ & $\mathrm{C}-\mathrm{H}$ bending/ $\mathrm{CO}_{3}^{-}$ & organic/carbonate ion & aliphatic/remaining carbonates \\
\hline $1500-1450$ & $\mathrm{C}=\mathrm{C}$ stretching & organic & aromatic rings \\
\hline 1585 & $\mathrm{COO}^{-}$antisym stretch & organic & carbox. acid salts \\
\hline$\sim 1600$ & $\mathrm{C}=\mathrm{C}$ ring stretch & organic & aromatic rings \\
\hline $1714-1700$ & $\mathrm{C}=\mathrm{O}$ stretch & organic & ketone/carboxylic acid \\
\hline $3000-2800$ & $\mathrm{CH}_{2} ; \mathrm{CH}_{3}$ sym and asym stretch & organic & aliphatic hydrocarbons \\
\hline $3600-2400$ (centered at 3250) & $\mathrm{OH}$ stretch & organic & alcohols, carbox. acids, phenols \\
\hline
\end{tabular}

Table 6. Aliphatic column density $\left(N[3.4]=N\left[\mathrm{CH}_{2}\right]+N\left[\mathrm{CH}_{3}\right]\right.$, see Table 7) in the region $2800-3000 \mathrm{~cm}^{-1}$ and silicate column density $(N[10])$ around $1000 \mathrm{~cm}^{-1}$ ) of the IDPs after the HF treatment, calculated after a baseline correction. See text for error estimates.

\begin{tabular}{cccccc}
\hline \hline IDP & area [3.4] & area [10] & $\begin{array}{c}N[3.4] \\
\left(\text { aliph C atom/cm } / \mathrm{cm}^{2}\right)\end{array}$ & $\begin{array}{c}N[10] \\
\left(\mathrm{Si} \text { atoms } / \mathrm{cm}^{2}\right)\end{array}$ & $\begin{array}{c}\text { SiO remaining } \\
\%\end{array}$ \\
\hline W7116B-N & 0.20 & 0.9 & $2.6 \times 10^{16}$ & $1.1 \times 10^{16}$ & 40 \\
W7116B-Y & 0.33 & 5.1 & $4.2 \times 10^{16}$ & $5.9 \times 10^{16}$ & 98 \\
W7116B-U & 0.15 & 2.6 & $2.3 \times 10^{16}$ & $3.1 \times 10^{16}$ & 26 \\
L2036-E19 & 0.02 & 0.3 & ${ }^{*} 2.0 \times 10^{15}$ & $3.7 \times 10^{15}$ & 6 \\
L2021-K3 & 0.12 & 0.1 & $1.4 \times 10^{16}$ & $1.3 \times 10^{15}$ & 3 \\
L2021-K2 & 0.17 & 0.9 & $1.9 \times 10^{16}$ & $1.1 \times 10^{16}$ & 30 \\
L2011-F2 & 0.09 & 0.1 & $1.3 \times 10^{16}$ & $1.6 \times 10^{15}$ & 2 \\
\hline
\end{tabular}

* This value was obtained by integration of the whole $3.4 \mu \mathrm{m}$ feature, since the $\mathrm{CH}_{2}$ and $\mathrm{CH}_{3}$ peaks were not well resolved.

Table 7. Column densities of the asymmetric aliphatic peaks in the region $2800-3000 \mathrm{~cm}^{-1}$ present in the IDPs spectra after the HF treatment, and of the IRS 7 source of the Galactic Center. The estimated error of these values, due to spectral noise and the quality of the Gaussian fit, is $\sim 10 \%$.

\begin{tabular}{cccccc}
\hline \hline IDP & $\begin{array}{c}\text { area }\left[\mathrm{CH}_{2}\right] \text { asym } \\
\sim 2922 \mathrm{~cm}^{-1}\end{array}$ & $\begin{array}{c}N\left[\mathrm{CH}_{2}\right] \\
\left(\mathrm{groups} / \mathrm{cm}^{2}\right)\end{array}$ & $\begin{array}{c}\text { area }\left[\mathrm{CH}_{3}\right] \mathrm{asym} \\
\sim 2958 \mathrm{~cm}^{-1}\end{array}$ & $\begin{array}{c}N\left[\mathrm{CH}_{3}\right] \\
\left(\mathrm{groups} / \mathrm{cm}^{2}\right)\end{array}$ & $N\left[\mathrm{CH}_{2}\right] / N\left[\mathrm{CH}_{3}\right]$ \\
\hline L2036-E19 & - & - & - & - & - \\
L2021-K3 & 0.039 & $1.1 \times 10^{16}$ & 0.014 & $2.6 \times 10^{15}$ & 4.2 \\
L2021-K2 & 0.059 & $1.6 \times 10^{16}$ & 0.016 & $2.9 \times 10^{15}$ & 5.5 \\
L2011-F2 & 0.036 & $1.0 \times 10^{16}$ & 0.017 & $3.2 \times 10^{15}$ & 3.1 \\
W7116B-N & 0.071 & $1.9 \times 10^{16}$ & 0.037 & $6.8 \times 10^{15}$ & 2.8 \\
W7116B-Y & 0.121 & $3.3 \times 10^{16}$ & 0.050 & $9.2 \times 10^{15}$ & 3.6 \\
W7116B-U & 0.062 & $1.7 \times 10^{16}$ & 0.033 & $6.1 \times 10^{15}$ & 2.8 \\
Average & & & & & 3.7 \\
\hline IRS 7 & 9.64 & $2.6 \times 10^{18}$ & 6.68 & $1.2 \times 10^{18}$ & 2.2 \\
\hline
\end{tabular}

anhydrous and hydrated IDPs contain roughly similar concentrations of aliphatic hydrocarbons. Both observations suggest that the aliphatic $\mathrm{C}-\mathrm{H}$ bonding in the two types of particles is similar.

\subsection{The $C=O$ carbonyl group at $\sim 1700 \mathrm{~cm}^{-1}$}

Some IDPs (see Table 8) show a peak around $1700 \mathrm{~cm}^{-1}$, that is attributed to the carbonyl stretch. This peak is not clearly present in the spectra taken before the HF treatment. However, after the acid etching the peak becomes clear and sharp in most of the particles, suggesting that this peak was partially masked by the $\mathrm{H}-\mathrm{O}-\mathrm{H}$ bending feature at $1660 \mathrm{~cm}^{-1}$, characteristic of the structural water. The comparison of $N[3.4]$ (Table 6) to $N[1700]$ (Table 8) shows that the number of $\mathrm{C}=\mathrm{O}$ bonds is for some particles only slightly lower than the number of $\mathrm{C}$ atoms in aliphatic chains. Based on its position $\left(\sim 1712 \mathrm{~cm}^{-1}\right)$, the carbonyl group can be attributed to either a ketone or a carboxylic acid. In order to find out whether this infrared absorption is produced by a ketone or by a carboxylic acid, we have compared our spectra with the spectra of a representative compound of each of these two groups: for ketones we used 


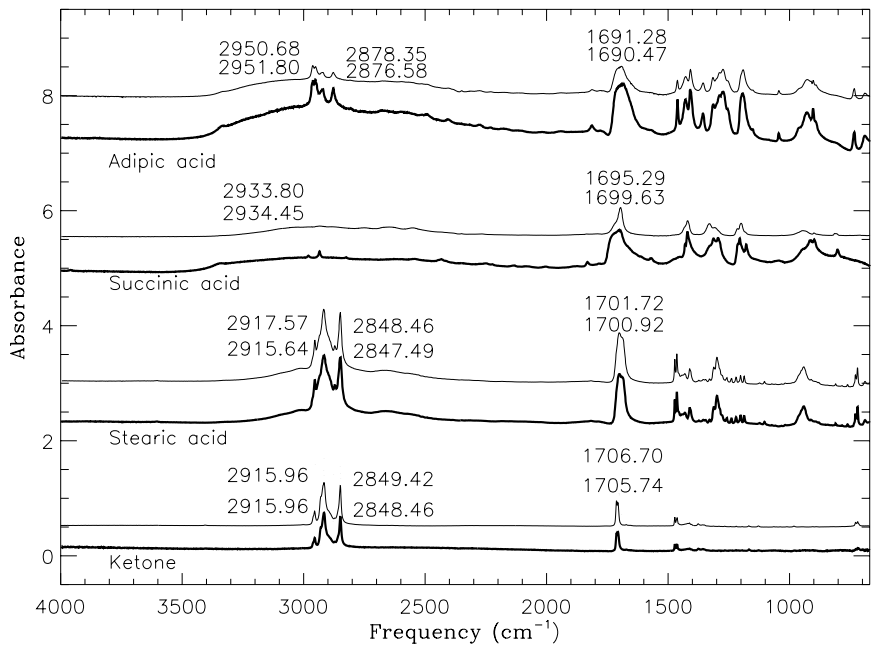

Fig. 6. Spectra of the standards 2-hexadodecanone, stearic, succinic and adipic acids before (solid line) and after (dashed line) HF treatment.

2-hexadodecanone; for carboxylic acids we used stearic, succinic, and adipic acids (Fig. 6). We exposed the standards to the same HF treatment used for the other samples, in order to test if the features at $1700 \mathrm{~cm}^{-1}$ and between $3000-2800 \mathrm{~cm}^{-1}$ change in profile, position or height after the acid treatment. It appeared that the hydrofluoric acid did not affect significantly any of the compounds used as standards, because both the peaks at $1700 \mathrm{~cm}^{-1}$ and around $3000 \mathrm{~cm}^{-1}$ have similar band shapes and positions after the HF treatment. For adipic and succinic acid there is a decrease in the absorbance, probably due to wash-out. These observations support the idea that the feature at $1700 \mathrm{~cm}^{-1}$ observed in some IDPs belongs to a carbonyl group and is not the result of a shift of a pre-existing peak after the HF treatment.

We compared some spectra of IDPs (W7116B-N; L2021-K3) after the acid etching to the two standards of ketone and carboxylic acid (Fig. 7). The precise position of the carbonyl group in the IDPs lies between 1706 and $1712 \mathrm{~cm}^{-1}$, which best matches the position of the carbonyl in a ketone. However, these IDPs also show a very broad band between $3500-2300 \mathrm{~cm}^{-1}$, which is characteristic of the $\mathrm{OH}$ stretch in carboxylic acids. Therefore, in this peak there is probably also some contribution from carboxylic acids. These features are found in both anhydrous and hydrated IDPs, suggesting that the presence of carboxylic acids and ketones is not silicate dependent.

\subsection{Carboxylic acid salts}

The spectrum of particle L2021-K2 before hydrolysis (Fig. 2) is particularly interesting. It displays two bands at 1583 and $1376 \mathrm{~cm}^{-1}$, which are compatible in shape and position with the $\mathrm{COO}^{-}$anti-symmetric and symmetric stretchings of ammonium salts of carboxylic acids. The spectrum after hydrolysis (Fig. 5) displays the same peaks, although their absorbance has decreased and they are blended with other peaks. A new peak appears at $1691 \mathrm{~cm}^{-1}$, which is probably due to carboxylic

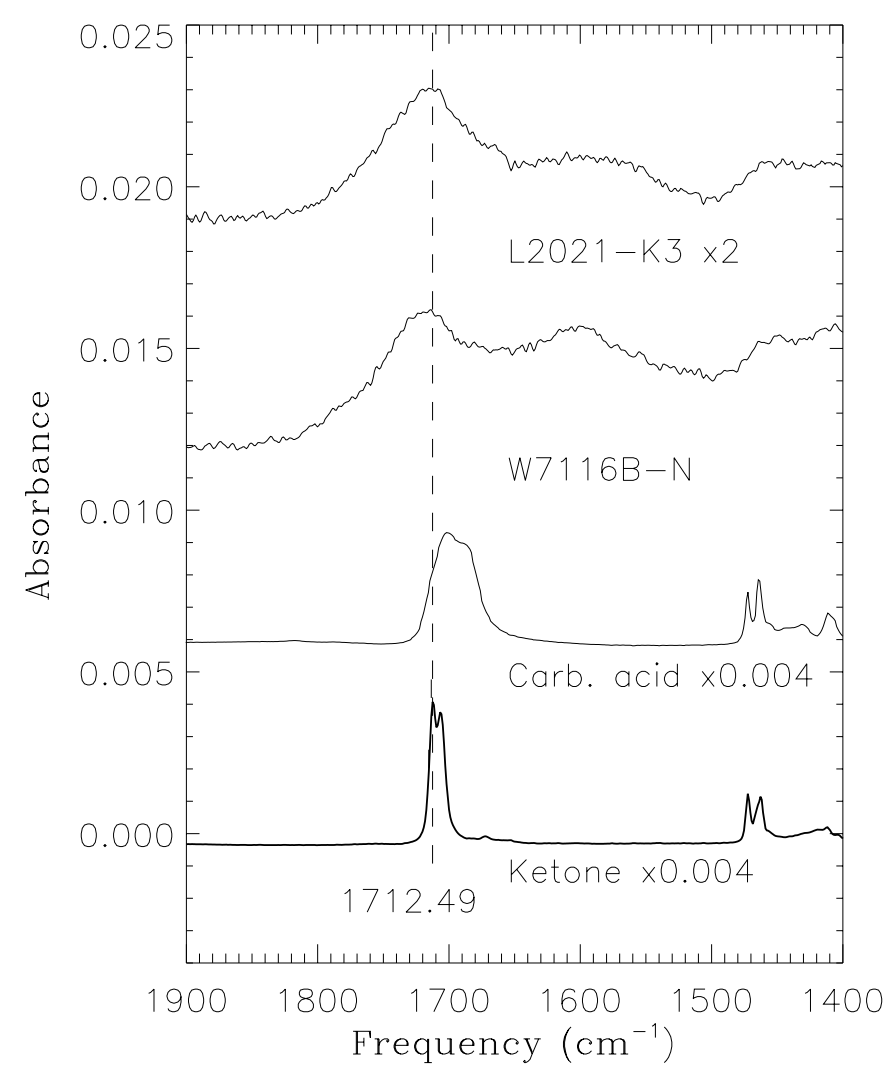

Fig. 7. Spectral region near $1700 \mathrm{~cm}^{-1}$ for the standards 2-hexadodecanone and stearic acid and their comparison with W7116B-N and L2021-K3 post HF particles.

acids. This supports the presence of carboxylic acid salts, since the hydrolysis of these compounds would lead to the formation of carboxylic acids by the substitution of the cation in the salt $\left(\mathrm{NH}_{4}^{+}\right.$for ammonium salts) by a $\mathrm{H}$. The comparison of $N$ [3.4] (Table 6) to $N$ [1585] (Table 8) shows that for particle L2021-K2 the number of $\mathrm{COO}^{-}$groups is double the number of aliphatic $\mathrm{C}$ atoms. Two other particles, L2011-F2 and W7116B-Y, might also contain carboxylic acid salts (see Table 8).

\subsection{Aromatics}

The relatively weak $\mathrm{CH}$ stretch band of aromatic hydrocarbons at $\sim 3030 \mathrm{~cm}^{-1}$ is not observed in the samples studied. A better tracer of aromatics is the band at $1600 \mathrm{~cm}^{-1}$ (ring stretch), as this band has a much larger intrinsic absorption than the $3030 \mathrm{~cm}^{-1}$ band and does not depend on the degree of hydrogenation of the aromatics. Three IDPs (L2021-K3, W7116B-U and $\mathrm{W} 7116 \mathrm{~B}-\mathrm{N}$ ) present a feature centered at $1600 \mathrm{~cm}^{-1}$, which could be due to an aromatic component.

The aromatic peaks observed in these IDPs could be attributed to PAHs, since PAHs have already been detected in some IDPs using other analytical techniques (Clemett et al. 1993). Assuming a band strength of $2 \times 10^{-19} \mathrm{~cm} \mathrm{C}$ atom $^{-1}$ (for average neutral PAHs, Schutte et al. 1998) these IDPs would have between $\sim 78$ and 114 times more carbon in aromatic than in aliphatic form. The other IDPs, however, do not show clear 
Table 8. Integration of other organic peaks present in the spectra after the HF treatment.

\begin{tabular}{|c|c|c|c|c|c|c|c|c|}
\hline IDP & $\begin{array}{l}\text { area[3600-2400] } \\
\text { carb. ac./hyd. sil. }^{a}\end{array}$ & $\begin{array}{c}\text { [3600-2400] } \\
\text { OH cm }{ }^{-2} \\
\text { carb. ac./hyd. sil. }{ }^{a}\end{array}$ & $\begin{array}{l}\text { area[ 1700] } \\
\text { carb. ac./ket. }{ }^{b}\end{array}$ & $\begin{array}{c}N[\sim 1700] \\
\mathrm{C}=\mathrm{O} \mathrm{cm} \mathrm{cm}^{-2} \\
\text { carb. ac./ket. }{ }^{b}\end{array}$ & $\begin{array}{c}\text { area[ 1600] } \\
\operatorname{arom}^{c}\end{array}$ & $\begin{array}{c}{[\sim 1600]} \\
\mathrm{C} \mathrm{\textrm {cm } ^ { - 2 }} \\
\text { arom. }^{c}\end{array}$ & $\begin{array}{l}\text { area }[\sim 1585] \\
\text { carb. ac. salt }{ }^{d}\end{array}$ & $\begin{array}{c}N[\sim 1585] \\
\mathrm{COO}^{-} \mathrm{cm}^{-2} \\
\text { carb. ac. salt }{ }^{d}\end{array}$ \\
\hline L2036-E19 & - & - & - & - & - & - & - & - \\
\hline L2021-K3 & 0.67 & $5.5 \times 10^{15}$ & 0.16 & $1.2 \times 10^{16}$ & 0.097 & $1.9 \times 10^{17}$ & - & - \\
\hline L2021-K2 & 2.21 & $1.8 \times 10^{16}$ & - & - & - & - & 0.98 & $3.8 \times 10^{16}$ \\
\hline L2011-F2 & 0.79 & $6.5 \times 10^{15}$ & 0.08 & $5.8 \times 10^{15}$ & - & - & 0.08 & $3.1 \times 10^{15}$ \\
\hline W7116B-N & 1.37 & $1.1 \times 10^{16}$ & 0.24 & $1.8 \times 10^{16}$ & 0.26 & $5.0 \times 10^{17}$ & - & - \\
\hline W7116B-Y & 1.11 & $9.1 \times 10^{15}$ & 0.12 & $8.9 \times 10^{15}$ & - & - & 0.19 & $7.3 \times 10^{15}$ \\
\hline W7116B-U & 0.41 & $3.3 \times 10^{15}$ & 0.14 & $1.0 \times 10^{16}$ & 0.18 & $3.5 \times 10^{17}$ & - & - \\
\hline
\end{tabular}

${ }^{a}$ This band will have a contribution of the $\mathrm{OH}$ stretch in hydrated silicates, and a contribution of the $\mathrm{OH}$ str. in carboxylic acids. For calculation of $N[3600-2400]$, we used $A=2.8 \times 10^{-16} \mathrm{~cm}(\mathrm{OH} \text { bond })^{-1}$ for carboxylic acids, see Table 3 .

${ }^{b}$ The band is probably due to the $\mathrm{C}=\mathrm{O}$ stretch of ketones with a contribution of carboxylic acids. For calculation of $N[\sim 1700]$, we used $A=3.2 \times 10^{-17} \mathrm{~cm}(\mathrm{CO} \text { bond })^{-1}$ for carboxylic acids. The column density using the band strength of ketones is 2.7 times larger, see Table 3 . ${ }^{c}$ Using $A=1.2 \times 10^{-18} \mathrm{~cm}(\mathrm{C} \text { atom })^{-1}$, for naphtalene in Ar matrix, see Table 3 .

${ }^{d}$ Using $A=6.0 \times 10^{-17} \mathrm{~cm}\left(\mathrm{COO}^{-}\right)^{-1}$, see Table 3. The values given for L2021-K2 correspond to the spectrum before HF treatment, as the salt is expected to be affected by hydrolysis. Indeed, for this particle, area[ $\sim 1585]$ decreased by $\sim 25 \%$ upon hydrolysis.

peaks around $1600 \mathrm{~cm}^{-1}$, indicating that the amount of aromatic components in our IDPs is very variable.

\subsection{The extraterrestrial origin of organics in IDPS}

It could be argued that some of the organic peaks observed during these analyses are indeed contamination that could have come from several sources: silicone oil, hexane used to clean it, the KRS-5 window, the terrestrial atmosphere. In order to investigate the possibility of contamination, we conducted analyses in several materials that we have used as blanks. In Fig. 8 we show the spectra of all the blanks used during this work.

a) Analysis of hexane: we measured the absorption spectrum of KRS-5 washed with a drop of hexane. Hexane rapidly evaporates and the measurement of the region where the drop was deposited did not reveal any feature in the $3.4 \mu \mathrm{m}$ region (see Fig. 8), proving that hexane does not leave any residual that might be a possible contamination. This analysis also demonstrates that the organic peaks observed in the IDP spectra are not associated with hexane, as expected, because of the quite inert nature of this solvent.

b) Analysis of KRS-5: we have acquired spectra for KRS-5 but a few particle-diameters off the particle, generally on the opposite side from the region where the background was taken, and determined the ratio of these and the original background. These spectra were all featureless, demonstrating that the absorption bands obtained in the spectra of our samples are not associated with the KRS-5 window.

c) Analysis of the silicone oil: we measured the absorption of silicone oil (extracted from the same collector from which the particles where extracted). The silicone oil presents several main peaks at 1260, 1095, 1020, 800 and $2963 \mathrm{~cm}^{-1}$. Generally, these peaks disappeared after the HF treatment, but sometimes traces of the oil remained, particularly visible at 1260 and $803 \mathrm{~cm}^{-1}$, the strongest bands. Except for the peak at $2963 \mathrm{~cm}^{-1}$, the other silicone oil peaks are away from the organic positions that we are investigating and thus they do not interfere with the recognition of organic features in the samples studied. The peak at $2963 \mathrm{~cm}^{-1}$ is a weak band ( $\sim 3$ times smaller than the 1260 band) that tends to disappear after the HF treatment (see example of particle L2011-F2, Figs. 2 and 5). Therefore, unless the $1260 \mathrm{~cm}^{-1}$ peak is huge, the organic features found in the IDPs studied cannot be attributed to the $2963 \mathrm{~cm}^{-1}$ silicone oil band.

d) Analysis of a piece of San Carlos olivine used as a reference: we investigated the ultra clean conditions of our setup by simulating the handling of a particle which did not contain any organics. For that we took a fragment of San Carlos olivine that was previously heated to $500{ }^{\circ} \mathrm{C}$ (in order to pyrolyze any organics that could be present on its surface) and put it inside a silicone oil drop for a few minutes. We then cleaned it with hexane and crushed it in a KRS-5 window. We analyzed and hydrolyzed this particle as we did with all the others. The spectrum of this particle before the HF treatment is shown in Fig. 8. The spectrum of the olivine after HF treatment is not available because the whole particle got etched. However, we have taken a spectrum of the region of the window where it was lying (not shown) where no features were found.

e) Analysis of two terrestrial particles: as a final test, we analyzed two particles (W7116-200, W7116B-K) classified as terrestrial from their fluorescence spectrum. These particles were taken from the same collector where the IDPs were extracted and they were prepared (cleaning, handling, dissolution, analysis) in the same manner as the IDPs. Their spectra, shown in Fig. 8, do not present any organic feature, suggesting that the organic peaks found in the IDPs are not associated to the collector or the atmosphere environment which they were collected from.

The analyses of these multiple blanks show that the organic features observed in the IDPs do not come from contamination during the cleaning, handling, analysis, HF treatment, etc. but are rather indigenous. Moreover, the extraterrestrial particle L2036-E19 can be considered a natural blank 


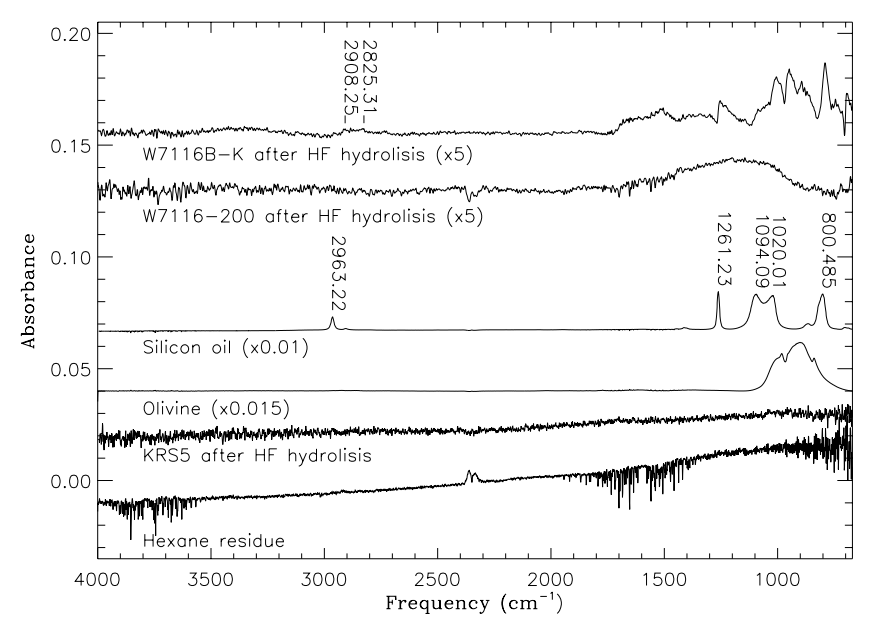

Fig. 8. Spectra of the materials used as blanks during this work: hexane; KRS-5; olivine; silicone oil; W7116-200; W7116B-K. For the latter two only the spectra after HF dissolution are shown.

since the $\mathrm{CH}$ signature observed before the $\mathrm{HF}$ treatment (Fig. 2, Table 4), practically disappeared after the HF treatment (Fig. 5), suggesting that its surface was contaminated with organics that were washed out by the HF. Flynn et al. (2003) also found three IDPs that did not present any obvious $\mathrm{C}-\mathrm{H}$ stretching absorption. If IDPs were contaminated, contamination would be expected to be spread over all the particles in the collector or to reach all the particles during handling. But this was not the case, as proved by the absence of aliphatic signatures in the two terrestrial particles W7116B-K and W7116-200. Thus, the presence of some organic-free particles supports that the organics we have found in the rest of the particles analyzed during this work are not associated with their terrestrial history or with contamination. The organics we see are therefore indigenous to the IDPs. Other works studying the isotopes of carbonaceous phases on IDPs also support that these phases are extraterrestrial in origin (Aléon et al. 2001, 2003; Keller et al. 2002).

\subsection{Comparison to previous studies}

\subsubsection{The hydrofluoric acid}

HF was used previously to dissolve the silicates in order to investigate the carbonaceous component of IDPs with FTIR (Flynn et al. 2002; Flynn et al. 2004). In these previous works, transmission electron microscope (TEM) sections of IDPs were hydrolyzed instead of the entire particle. Thus, if the organics are heterogeneously distributed throughout the particle, there is no way to obtain the right section for the analysis. This is the reason why in this work we have performed an in situ acid dissolution of the entire crushed particle. This presents the advantage that the silicates composing the particle are almost entirely dissolved, and thus that all the acid-insoluble carbonaceous matter of the particle is concentrated, improving the detection and resolution of the aliphatic bands. The HF treatment will be further discussed in Sect. 4.1.

\subsubsection{The remaining silicone oil}

Some IDP spectra present peaks at 1260, 1100 and $803 \mathrm{~cm}^{-1}$ which are consistent with the main peaks of silicone oil (see Fig. 8), indicating that the hexane in some cases does not entirely remove the silicone oil from the particle. In most cases, the remaining silicone oil is reduced or totally washed out with the HF (see Tables 1 and 5). However, in a few cases a tiny peak of silicone oil at $1260 \mathrm{~cm}^{-1}$ is still present. Flynn et al. (2002, 2004) also found some unremoved silicone oil in their samples, which suggests that this problem is not intrinsic to our method of etching but rather to the difficulty of completely removing the silicone oil by dissolution with organic solvents (hexane) or acids (HF).

To estimate the contribution of the silicone oil peaks to the areas of the integrated bands is not straightforward, as often the silicone oil peaks overlap with both the silicate and the organic features we are interested in. However, from integration of the $1260 \mathrm{~cm}^{-1}$ peak due to silicone oil, and using the relative area values of the silicone oil peaks as deduced from the standard spectrum of silicone oil shown in Fig. 8, we can give a rough estimate of the contribution of silicone oil to the observed areas of the silicate and aliphatic $\mathrm{CH}$-stretching bands, respectively in the 10 and $3.4 \mu \mathrm{m}$ regions. In particular, it was found that the area of the $2963 \mathrm{~cm}^{-1}$ band is 3 times smaller than the area of the $1260 \mathrm{~cm}^{-1}$ band, which made it possible to estimate roughly the contribution of silicone oil to the $3.4 \mu \mathrm{m}$ band. For both bands, this contribution is of the order of $10 \%$ or lower before hydrolisis, as for IDP W7116B-N, except for particles W7116B-Y and L2011-F2 which contain more silicone oil. After hydrolysis, little silicone oil remains in the spectra, except for particles W7116B-Y and W7116B-U (as observed by comparison of Figs. 1 and 2 with Figs. 4 and 5). The presence of silicone oil is only problematic for the determination of the $\mathrm{CH}_{2} / \mathrm{CH}_{3}$ ratio, as the position of the $2963 \mathrm{~cm}^{-1}$ silicone oil peak is expected to overlap with the $2958 \mathrm{~cm}^{-1}$ band $\left(\mathrm{CH}_{3}\right.$ asymmetric vibration). However, except for IDPs W7116B-Y and W7116B-U, the $\mathrm{CH}_{2} / \mathrm{CH}_{3}$ values after hydrolysis should not be affected, as little silicone oil remains.

\subsubsection{Analyses of blanks}

Some of the blanks (silicone oil, hexane and terrestrial particles) used during this work to control our results for contamination were also employed by Flynn et al. (2004). In this previous work, the analyses of blanks did not show any aliphatic signature for evaporated hexane or silicone oil. However, the terrestrial particles showed a weak $\mathrm{C}-\mathrm{H}$ absorption feature at $\sim 2960 \mathrm{~cm}^{-1}$ and positions near but not identical to those seen in their IDPs. This suggests that terrestrial contamination does not affect very much the FTIR analyses since the terrestrial C-H signatures have positions and strengths different from IDPs.

\subsubsection{Variability in the aliphatic fraction among IDPs}

The identification of organics in most of the particles we analyzed showed that both anhydrous and hydrated IDPs contain organics. This was also observed by Flynn et al. (2003, 2004) 
and Raynal et al. (2001). It was also observed that the positions of the aliphatic peaks around the $3.4 \mu \mathrm{m}$ region vary slightly from one particle to another. However, these variations are still in the ranges of the known $\mathrm{CH}_{2}$ and $\mathrm{CH}_{3}$ peak positions.

The column densities of the aliphatic peaks calculated after the HF treatment (Table 7) show that the aliphatic $\mathrm{CH}_{2}$ and $\mathrm{CH}_{3}$ groups are present in different quantities in each IDP, regardless of kind (anhydrous or hydrated). This observation was also made by Flynn et al. (2003) and suggests that some IDPs are richer in aliphatics than others. This also suggests that the aliphatic abundance is not dependent on the kind of silicate that constitutes the IDP particle.

\subsubsection{Diversity of organics in IDPs}

Besides aliphatic chains, some IDPs also have a carbonyl group $(\mathrm{C}=\mathrm{O})$. This functional group was identified in IDPs during previous studies using FTIR (Flynn et al. 2002; Flynn et al. 2003, 2004) and using carbon and oxygen XANES (Reffner et al. 1994; Keller et al. 2002; Flynn et al. 2003). These previous results corroborate our finding of a $\mathrm{C}=\mathrm{O}$ functional group in most of the IDPs studied.

\section{Discussion}

\subsection{The in situ HF dissolution}

The use of HF during this work made it possible to improve the recognition of several organic features. Indeed, diffusion is enhanced by the different diffraction indexes coming from both the silicates and the carbonaceous material present in the particle. Removal of the silicates reduces this effect.

The values of the silicate column densities $(N[10]$, see Tables 4 and 6) show that for all IDPs, except W7116B-Y, $N$ [10] clearly diminishes after the HF treatment (from about 2.5 to 50 times), showing that the in situ HF treatment is very efficient in dissolving the silicates. Furthermore, for most IDPs the bands in the $3000 \mathrm{~cm}^{-1}$ region are much better resolved, which allowed the calculation of the column densities corresponding to the $\mathrm{CH}_{2}$ and $\mathrm{CH}_{3}$ groups. Moreover, after the $\mathrm{HF}$ etching the bands in the $1000-1700 \mathrm{~cm}^{-1}$ region, such as the carbonyl peak and the $\mathrm{C}=\mathrm{C}$ bonds, are now visible, as there is little overlap with the bands due to silicates and other minerals. This has allowed the detection of a ketone and/or a carboxylic acid compound in most of the particles, and also of the presence of the $1600 \mathrm{~cm}^{-1}$ band ascribed to aromatics. Thus, the dissolution method with HF developed in this work has been very useful for improving the detection of several organic features in these particles. However, it is necessary to test the hydrolysis protocol with molecular standards, as there might be organic components that could be affected by the hydrolysis.

\subsection{Comparison with the acid extract of carbonaceous chondrites}

Several investigations of the acid extract of the carbonaceous chondrites Murchison and Orgueil have been performed to date
(Ehrenfreund et al. 1991; de Vries et al. 1993; Gardinier et al. 2000). All of them showed that both meteorites present four main peaks at 2960, 2925, 2875 and $2855 \mathrm{~cm}^{-1}$, with the latter two bands blended into one single band around $2865 \mathrm{~cm}^{-1}$. In addition, the aliphatic fraction of the carbonaceous chondrite Tagish Lake was also investigated (Matrajt et al. 2004) and it was found that the meteorite is dominated by long aliphatic chains, with a $\mathrm{CH}_{2} / \mathrm{CH}_{3}$ ratio of $\sim 7.3$. We have compared the spectra of our IDPs with the ones available from Murchison and Orgueil. This comparison shows that the band widths of the $3.4 \mu \mathrm{m}$ feature in IDPs are narrower than in the meteorites (by about $30-40 \mathrm{~cm}^{-1}$ ), suggesting that the aliphatic chains in IDPs are made of simpler compounds. Moreover, the $\mathrm{CH}_{2} / \mathrm{CH}_{3}=3.7$ ratio of the seven IDPs studied is larger than the value of $\sim 2$ corresponding to Murchison and Orgueil, but shorter than the value of $\sim 7$ corresponding to Tagish Lake. It can thus be concluded that the aliphatic chains in IDPs are longer (or less ramified) than in Murchison and Orgueil but shorter (or more ramified) than in Tagish Lake, showing that the aliphatic material in IDPs is different from that found in carbonaceous chondrites. This suggests that the carbonaceous material found in both kinds of objects was formed (or was processed) in different environments and/or under different conditions, and therefore has a different origin.

Flynn et al. (2004) and Keller et al. (2004) compared the carbonyl group present in Murchison to the one observed in IDPs and found that it has a similar position in both objects. The position of the carbonyl found in our IDPs is also similar to that of the carbonyl in Murchison, suggesting that both carbonaceous chondrites and IDPs have a fraction of oxygen-rich organics harboring a carbonyl functional group.

\subsection{Comparison to the $3.4 \mu \mathrm{m}$ region in the DISM}

To investigate the origin (molecular cloud material, diffuse interstellar material, ...) of the organic phases observed in these IDPs, we compared the acid extract of three of the IDPs, the W7116B-U, W7116B-Y and L2021-K2 particles, to the spectrum of the GC IRS 7 source, a probe of the diffuse interstellar medium (Fig. 9). This comparison clearly shows that the IDP spectra and the DISM spectrum are different in several significant ways. First, the IDP spectra are dominated by the $\mathrm{CH}_{2}$ peaks (at $\sim 2923$ and $2855 \mathrm{~cm}^{-1}$ ) whereas the DISM spectrum has $\mathrm{CH}_{2}\left(\right.$ at $\sim 2923 \mathrm{~cm}^{-1}$ ) and $\mathrm{CH}_{3}$ (at $\sim 2958 \mathrm{~cm}^{-1}$ ) peaks of similar strength, and, unlike for IDPs, the $\mathrm{CH}_{3}$ peak at $\sim 2865 \mathrm{~cm}^{-1}$ is clearly present. Indeed the computed average $\mathrm{CH}_{2} / \mathrm{CH}_{3}$ ratio for IDPs is 3.7 (see Table 7 and Sect. 3.2.2), suggesting that in the DISM the aliphatics are either more ramified or shorter than in the IDPs. Flynn et al. (2003) also found that the average ratio in their IDPs, ranging from $2.31 \pm 1.74$ in the hydrated IDPs to $2.46 \pm 1.32$ in the anhydrous IDPs, was higher than in the DISM. Second, the $3.4 \mu \mathrm{m}$ band in the IDP spectra is narrower than the band corresponding to dust absorption in the DISM, suggesting that the molecules in the IDPs are simpler. All together, the aliphatic component in the DISM is more complex and ramified than in IDPs, suggesting that the two carbonaceous compounds were formed in different 


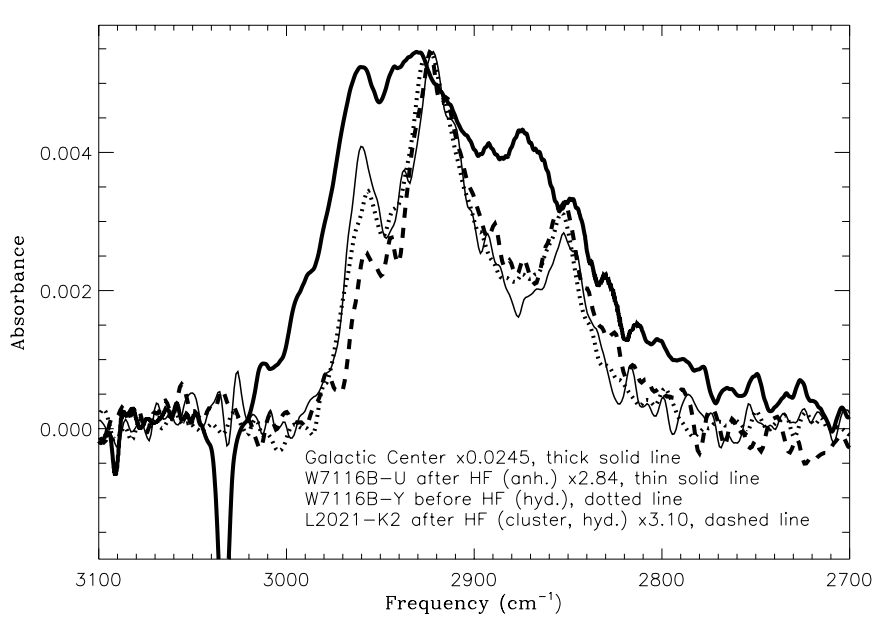

Fig. 9. Comparison of the spectrum of the particles W7116B-Y (hydrated) before HF, L2021-K2 (cluster, hydrated) after HF and W7116B-U (anhydrous) after HF to the IRS 7 source (Galactic Center). Only the $2800-3100 \mathrm{~cm}^{-1}$ region is shown. The Galactic Center source spectrum has been obtained from the Infrared Space Observatory (ISO) Data Center.

environments or under different conditions and at different times in the Universe.

It is worth noting that the L2021-K2 particle is a cluster particle (Table 2). Messenger (2000) found significant excesses of $\mathrm{D},{ }^{15} \mathrm{~N}$ and ${ }^{16} \mathrm{O}$ in cluster particles and deduced that these particles contained "preserved interstellar material" and therefore were the most primitive class of Solar System materials. However, the $3.4 \mu \mathrm{m}$ feature in cluster IDPs is similar to that of the other IDPs studied, and it is different from the $3.4 \mu \mathrm{m}$ feature of the diffuse interstellar medium. Therefore, the aliphatic hydrocarbons in cluster IDPs are not preserved diffuse interstellar materials but rather materials that formed in a different environment from the diffuse interstellar medium, such as dense clouds, or materials that were re-processed in the Solar System.

\subsection{Origin of the $3.4 \mu \mathrm{m}$ feature in IDPs. Why is this band different from the one observed in the DISM?}

\subsubsection{Alteration by the HF treatment?}

It could be argued that the ratios measured during this study in the aliphatics of IDPs have been altered during the experimental process of etching with HF. We have shown that, after etching, several organic peaks that were masked by the silicate peaks are now visible or better resolved, showing that the etching is helping to detect more organic material.

In Fig. 3 are shown the $\mathrm{CH}_{2}$ and $\mathrm{CH}_{3}$ peaks (before and after the HF treatment) corresponding to the region $3000-2800 \mathrm{~cm}^{-1}$. The peaks in the spectra before the HF etching (dashed line) are not well resolved in most of the particles. However, particle W7116B-Y shows an intense and wellresolved $3.4 \mu \mathrm{m}$ feature previous to hydrolysis. Comparison of this band before and after HF treatment shows that the shape, band positions and $\mathrm{CH}_{2} / \mathrm{CH}_{3}$ ratio are not modified by the hydrolysis. Furthermore, in Fig. 6 are shown the spectra of four organic standards before and after having been treated with HF. In this figure the peaks in the region $3000-2800 \mathrm{~cm}^{-1}$ are well resolved and it is clear that they have not changed (neither in position, nor in relative height) after the HF treatment. We can conclude that the $\mathrm{HF}$ etching is not altering the $\mathrm{CH}_{2} / \mathrm{CH}_{3}$ ratios measured, suggesting that these ratios in IDPs are originally different from that observed in the DISM.

\subsubsection{Alteration by heating during atmospheric entry?}

It could also be argued that the $\mathrm{CH}_{2} / \mathrm{CH}_{3}$ ratios measured in these IDPs are different from the diffuse ISM because of the heating during the atmospheric entry of these particles. The high temperatures to which these particles are exposed during their entry would have altered or evaporated some molecules, changing the overall $\mathrm{CH}_{2} / \mathrm{CH}_{3}$ ratio of the remaining organic material. Models of atmospheric entry heating of IDPs suggest that IDPs are heated for a few seconds to 600-800 K during their atmospheric entry (Flynn 1989). But there must be a gradient in the temperature to which the particle is really exposed, allowing the survival of volatile compounds, since ketones and carboxylic acids are found in IDP matrices, as we have demonstrated in the present work and as has been demonstrated during other studies (Flynn et al. 2003, 2004). We have also demonstrated the presence of indigenous aliphatics in the matrices of these IDPs, and aliphatics do not survive at temperatures above 800-1000 K (Dartois, unpublished results). Wdowiak et al. (1988) performed heating experiments at several temperatures up to $800 \mathrm{~K}$ of the acid insoluble residue of the Orgueil carbonaceous chondrite. These experiments demonstrated that the evaporating temperature of the compounds forming the bands in the $3000 \mathrm{~cm}^{-1}$ region lies in the range $700-800 \mathrm{~K}$. In these experiments, it was clearly shown that the peaks in this region do not change shape or relative height (see Fig. 1 of Wdowiak et al. 1988), they only decrease in size with temperature because of evaporation, suggesting that the ratio $\mathrm{CH}_{2} / \mathrm{CH}_{3}$ is not affected by temperature. Therefore, it is unlikely that the ratio $\mathrm{CH}_{2} / \mathrm{CH}_{3}$ measured in the IDPs was altered by atmospheric entry heating. However, more pulse-heating experiments are needed to completely rule out this possibility.

\subsubsection{Presence of PAHs?}

PAHs (Polycyclic Aromatic Hydrocarbons) are present in IDPs (Allamandola et al. 1987; Clemett et al. 1993). Reactions transforming these PAHs could lead to the formation of $\mathrm{CH}_{2}$ side groups. For example, the UV photolysis of PAHs produces partially hydrogenated aromatic hydrocarbons (Bernstein et al. 1999, 2002) and alkylated PAHs (Mahajan et al. 2003). However, a quantification of the PAH abundances in IDPs does not exist to date. We found that three out of the seven IDPs showed a band at $\sim 1600$ characteristic of aromatics, which indicates that they contain at most $\sim 78-114$ times more carbon in aromatic than in aliphatic form (see Sect. 3.5). However, the other IDPs did not present a band at this position. Moreover, unlike aliphatic compounds, aromatic hydrocarbons are not an 
ubiquitous component of the dust in the general DISM (Chiar et al. 2000). Therefore, the mechanism described above would take place only rarely and thus it is unlikely that the $3.4 \mu \mathrm{m}$ feature measured in these IDPs would originate from aromatic precursors.

Alternatively, the presence of $\mathrm{H}_{n}$-PAHs (PAHs that have a few additional hydrogen atoms attached, Schutte et al. 1993; Bernstein et al. 1996) in IDPs, would lead to an increase on strength of the features characteristic of the symmetric and asymmetric $\mathrm{C}-\mathrm{H}$ stretching vibrations of the $\mathrm{CH}_{2}$ groups (Bernstein et al. 1996). However, these features in $\mathrm{H}_{n}$-PAHs have their positions slightly shifted from the normal position in aliphatics, and usually fall in the vicinity of 2940 and $2850 \mathrm{~cm}^{-1}$ (Bernstein et al. 1996). In the IDPs we have studied during this work the symmetric and asymmetric positions of the $\mathrm{CH}_{2}$ groups are well defined and constant: 2850 and $2922 \mathrm{~cm}^{-1}$, respectively. Therefore, it seems unlikely that the $\mathrm{CH}_{2}$ features observed in IDPs contain a significant contribution from the $\mathrm{CH}_{2}$ features typical of $\mathrm{H}_{n}$-PAHs because the band near $2940 \mathrm{~cm}^{-1}$ is not observed (see Fig. 3). The absence of such a band also suggests that if $\mathrm{H}_{n}$-PAHs are present in these IDPs, they are not linked to the aliphatic molecules. Therefore, it is unlikely that the ratio $\mathrm{CH}_{2} / \mathrm{CH}_{3}$ measured in the IDPs is different from the one observed in the DISM by the presence of PAHs. The observed difference must be attributed to the fact that the carbonaceous material found in these IDPs was formed in an environment different from the diffuse interstellar medium.

\section{Conclusions and perspectives}

In this work we have analyzed seven interplanetary dust particles (IDPs) using an infrared microscope. We have developed an in situ extraction method based on the dissolution of the silicate phases with hydrofluoric acid, which allowed the organic phases to be better detected. We have found that six of the seven particles studied present a well resolved $3.4 \mu \mathrm{m}$ feature which corresponds to aliphatic chains. Most of the particles analyzed (L2021-K3, L2011-F2, W7116B-N, W7116B-Y and $\mathrm{W} 7116 \mathrm{~B}-\mathrm{U})$ also presented a carbonyl group around $1700 \mathrm{~cm}^{-1}$, which appears to be due to ketones with some contribution of carboxylic acids. Some particles (L2021-K3, W7116B-U and W7116B-N) show a band around $1600 \mathrm{~cm}^{-1}$, which can be attributed to aromatics. We calculated the column densities of functional groups and aromatics in the IDPs studied. We determined the column densities of the asymmetric $\mathrm{CH}_{3}$ and $\mathrm{CH}_{2}$ peaks in our IDPs and we calculated their $\mathrm{CH}_{2} / \mathrm{CH}_{3}$ ratios using a Gaussian fit, obtaining an average of 3.7. We did not observe a significant difference between the hydrated and the anhydrous IDPs. Using the same fit we also re-determined the $\mathrm{CH}_{2} / \mathrm{CH}_{3}$ ratio in the GC IRS 7 interstellar object, and found a value of 2.2. The comparison of this value to the one obtained for the IDPs indicated that the aliphatic chains in the IDPs are longer (or less ramified) than in the diffuse ISM. Given that the $3.4 \mu \mathrm{m}$ band of organics in the diffuse ISM is significantly broader than the same band in IDPs, we conclude that the organics in IDPs are simpler than the organics present in the diffuse ISM, suggesting that the carbonaceous compound found in these IDPs has been formed in a different environment from the diffuse ISM.

Studies concerning the origin and evolution of the organic fraction in IDPs are currently in process.

Acknowledgements. We acknowledge the CNES-INSU who provided post-doctoral support for G.M., and the Marie Curie fellowship program who provided post-doctoral support for G.M.M.C. We thank P. Dumas for his assistance during the FTIR analyses. We acknowledge the NASA curation facility (in particular M. Zolensky) who provided the samples used during this study.

\section{References}

Aléon, J., Engrand, C., Robert, F., \& Chaussidon, M. 2001, Geochim. Cosmochim. Acta, 65, 4399

Aléon, J., Robert, F., Chaussidon, M., \& Marty, B. 2003, Geochim. Cosmochim. Acta, 67, 3773

Allamandola, L., Sandford, S., \& Wopenka, B. 1987, Science, 237, 56

Bernstein, M. P., Elsila, J. E., Dworkin, J. P., et al. 2002, ApJ, 576, 1115

Bernstein, M. P., Sandford, S. A., \& Allamandola, L. J. 1996, ApJ, 472, L127

Bernstein, M. P., Sandford, S. A., Allamandola, L. J., et al. 1999, Science, 283, 1135

Bradley, J. P., Brownlee, D. E., \& Fraundorf, P. 1984, Science, 223, 56

Bradley, J. P., Sandford, S. A., \& Walker, R. M. 1988, in Meteorites and the early Solar System, ed. J. F. Kerridge, \& M. S. Matthews (Tucson: The University of Arizona Press), 861

Bradley, J. P., Keller, L. P., Snow, T. P., et al. 1999, Science, 285, 1716

Brownlee, D. E. 1978, in Protostars and Planets: Studies of Star Formation and of the Origin of the Solar System, 134

Brownlee, D. E., Joswiak, D. J., Bradley, J. P., Gezo, J. C., \& Hill, H. G. M. 2000, Lunar Planet. Sci. (Abs), 31, 1921

Chiar, J. E., Tielens, A. G. G. M., Whittet, D. C. B., et al. 2000, ApJ, 537, 749

Clemett, S., Maechling, C., Zare, R., Swan, P., \& Walker, R. 1993, Science, 262, 721

Clemett, S., Messenger, S., Keller, L., \& Zare, R. 1999, Lunar Planet. Sci., XXX, CDROM \# 1783

Dartois, E., Marco, O., Muñoz-Caro, G., et al. 2004, A\&A, 423, 549

de Vries, M. S., Reihs, K., Wendt, H. R., et al. 1993, Geochim. Cosmochim. Acta, 57, 933

Ehrenfreund, P., Robert, F., D’Hendencourt, L., \& Behar, F. 1991, A\&A, 252, 712

Flynn, G. J. 1989, Icarus, 77, 287

Flynn, G., Keller, L., \& Miller, M. 1998, Lunar Planet. Sci., 29, 1157

Flynn, G. J., Keller, L. P., Joswiak, D., \& Brownlee, D. E. 2002, Lunar Planet. Sci. (Abs), 1320

Flynn, G. J., Keller, L. P., Feser, M., Wirick, S., \& Jacobsen, C. 2003, Geochim. Cosmochim. Acta, 67, 4791

Flynn, G. J., Keller, L., Jacobsen, C., \& Wirick, S. 2004, Adv. Space Res., 33, 57

Fraundorf, P. 1981, Geochim. Cosmochim. Acta, 45, 915

Gardinier, A., Derenne, S., Robert, F., et al. 2000, Earth and Planetary Science Letters, 184, 9

Jaeger, C., Molster, F. J., Dorschner, J., et al. 1998, A\&A, 339, 904

Keller, L. P., \& Flynn, G. J. 2003, Lunar Planet. Sci. (Abs), 34, 1903

Keller, L. P., Hony, S., Bradley, J. P., et al. 2002, Nature, 417, 148

Keller, L. P., Messenger, S., Flynn, G. J., et al. 2004, Geochim. Cosmochim. Acta, 68, 2577

Koike, C., Shibai, H., \& Tuchiyama, A. 1993, MNRAS, 264, 654 
Mahajan, T. B., Elsila, J. E., Deamer, D. W., \& Zare, R. N. 2003, Orig. Life Evol. Biosph., 33, 17

Matrajt, G., Borg, J., Raynal, P., et al. 2004, A\&A, 416, 983

Messenger, S. 2000, Nature, 404, 968

Molster, F. J., Bradley, J. P., Sitko, M. L., \& Nuth, J. A. 2001, Lunar Planet. Sci. (Abs), 32, 1391

Molster, F. J., Demyk, A., D’Hendecourt, L., \& Bradley, J. P. 2003, Lunar Planet. Sci. (Abs), 34, 1148

Pendleton, Y. J., Sandford, S. A., Allamandola, L. J., Tielens, A. G. G. M., \& Sellgren, K. 1994, ApJ, 437, 683

Raynal, P. I., Quirico, E., Borg, J., et al. 2000, Planet. Space Sci., 48, 1329

Raynal, P. I., Quirico, E., Borg, J., \& D'Hendecourt, L. 2001, Lunar Planet. Sci. (Abs), 32, 1341
Reffner, J., Carr, G. L., Sutton, S., Hemley, R. J., \& Williams, G. P. 1994, Synchrotron Radiation News, 7, 30

Robert, F., \& Epstein, S. 1982, Geochim. Cosmochim. Acta, 46, 81

Sandford, S. A., \& Walker, R. M. 1985, ApJ, 291, 838

Sandford, S. A., Allamandola, L. J., Tielens, A. G. G. M., et al. 1991, ApJ, 371, 607

Schutte, W. A., Tielens, A. G. G. M., \& Allamandola, L. J. 1993, ApJ, 415, 397

Schutte, W. A., van der Hucht, K. A., Whittet, D. C. B., et al. 1998, A\&A, 337, 261

Smith, J., \& Kaplan, I. 1970, Science, 167, 1367

Wdowiak, T. J., Flickinger, G. C., \& Cronin, J. R. 1988, ApJ, 328, L75

Wexler, A. 1967, Appl. Spectrosc. Rev., 1, 29 